Publ. RIMS, Kyoto Univ.

32 (1996), 117-156

\title{
A Discrete Model of the Integer Quantum Hall Effect
}

\author{
By \\ Paul J. McCANN ${ }^{*}$ and Alan L. CAREY ${ }^{*}$
}

\begin{abstract}
A discrete model of the integer quantum Hall effect is analysed via its associated $C^{*}$ algebra. The relationship with the usual continuous models is established by viewing the observable algebras of each as both twisted group $C^{*}$-algebras and twisted cross products. A Fredholm module for the discrete model is presented, and its Chern character is calculated.
\end{abstract}

\section{$\S 1$. Introduction}

The discovery of the integer quantum Hall effect has prompted a wealth of theoretical speculation about the origin of the spectacular accuracy with which the Hall conductance is quantized. This paper presents a simple lattice model of the quantum Hall effect that generates much of the information arising from more complex models. This lattice model of the quantum Hall effect is often used as the discrete analogue of the Landau Hamiltonian in the physics literature, and the analysis of the model often requires restricting to rational values of the magnetic flux. It is here extended and recast to fit into the $C^{*}$-algebraic framework, a development that allows (in \& 3) the Hall conductance to be calculated for all real values of flux. The analysis of the expression for the conductance makes its stability with respect to small changes in magnetic field evident, for it is found to be the Chern number associated with the Fermi projection (when the latter lies in a gap of the spectrum of the discrete Hamiltonian). We display the equivalence with the formula found for rational flux in the physics literature by using an explicit representation of the algebra of observables.

The Hall effect is often modelled by considering electrons moving on a plane under the influence of a perpendicular magnetic field and a periodic potential. We show in $\S 2$ that for both this model and the discrete model mentioned above the

Communicated by H. Araki, April 25, 1995.

1991 Mathematics Subject Classifications: 20G45, 47C15, 46L87.

* Department of Pure Mathematics, University of Adelaide, Adelaide, 5005, Australia. 
algebra of observables can be written as a twisted group $C^{*}$-algebra. This enables us to establish a precise relationship between the discrete and continuous models in three ways, each of which provides information and insight. First we use the theory developed by Mackey to display the equivalence of their representation theories: any factor representation of the continuous algebra of observables is induced from a factor representation of the discrete algebra. Second, the algebras are recast as twisted cross products and this is used to calculate a series of isomorphisms culminating in the identification of the algebra of observables in the continuous model as simply the stabilized form of that of the discrete model. This implies in particular the third connection: they are Morita equivalent, which implies that their $K$-theory is identical.

The main results are contained in $\S 4$ where we present a Fredholm module for the discrete model that plays a similar role to the well known module that Bellissard produced for the continuous model. The Chern character for the Fredholm module over the algebra for the discrete model is calculated explicitly, and we show that it coincides with the Chern character associated with a familiar cycle over the rotation algebra (this is the analogue for the discrete model of the Connes-Kubo formula). We also note that the Fredholm module allows the identification of the Hall conductance as the index of a Fredholm operator.

\section{§2. Preliminaries}

\section{$\S 2.1$. The Continuous Model}

We begin by reviewing the usual model. We consider the Hamiltonian on $L^{2}\left(\mathbb{R}^{2}\right)$ that represents electrons moving in a plane with a periodic potential and a magnetic field perpendicular to the plane:

$$
H=1 / 2\left(-\partial_{x}^{2}+\left(-i \partial_{y}-\alpha x\right)^{2}\right)+V(x, y)
$$

where the potential $V$ is required to satisfy the periodicity requirements,

$$
V(x+1, y)=V(x, y+1)=V(x, y) \text {, }
$$

and $\alpha$ is the number of magnetic flux quanta through a unit cell. We've chosen the Landau gauge here, but other choices will arise (by necessity) later in this section, where they appear via cohomologous cocycles in twisted group $C^{*}$ algebras.

Note that the magnetic translation operators, defined by

$$
\left(U_{0} f\right)(r, s)=e^{2 \pi i \alpha s} f(r+1, s) \text { and }\left(U_{1} f\right)(r, s)=f(r, s+1) \text {, }
$$


for $f \in L^{2}\left(\mathbb{R}^{2}\right)$, are symmetries of the system under consideration, and so lie in the commutant of the algebra of observables. We define two twisted translations corresponding to the "momentum" operators $-i \partial_{x}$ and $-i \partial_{y}-\alpha x$ in the Hamiltonian given above by

$$
T_{x} f(r, s)=f(r+x, s) \text { and } \tilde{T}_{y} f(r, s)=e^{2 \pi i r \alpha y} f(r, s+y)
$$

and let $M_{\left(m_{1}, n_{1}\right)}$ denote the multiplication operator

$$
\left(M_{\left(m_{1}, n_{1}\right.} f\right)(r, s)=\exp \left(2 \pi i\left(m_{1} r+n_{1} s\right)\right) f(r, s),
$$

so that $\left\{M_{\left(m_{1}, n_{1}\right)}:\left(m_{1} n_{1}\right) \in \mathbb{Z}^{2}\right\}$ generates a representation of $C\left(\mathbb{T}^{2}\right)$ on $L^{2}\left(\mathbb{R}^{2}\right)$.

Consider now the product $T_{x_{1}} \tilde{T}_{y_{1}} M_{m_{1}, n_{1}} T_{x_{2}} \tilde{T}_{y_{2}} M_{m_{2}, n_{2}}$. Easy calculations give

$$
\begin{gathered}
M_{m_{1}, n_{1}} T_{x_{2}} M_{m_{1}, n_{1}}{ }^{-1}=\exp \left(-2 \pi i x_{2} m_{1}\right) T_{x_{2}} \\
M_{m_{1}, n_{1}} \tilde{T}_{y_{2}} M_{m_{1}, n_{1}}{ }^{-1}=\exp \left(-2 \pi i n_{1} y_{2}\right) \tilde{T}_{y_{2}} \\
T_{x_{2}}{ }^{-1} \tilde{T}_{y_{1}} T_{x_{2}}=\exp \left(-2 \pi i x_{2} y_{1} \alpha\right) \tilde{T}_{y_{1}} .
\end{gathered}
$$

Combining these we obtain

$$
T_{x_{1}} \tilde{T}_{y_{1}} M_{m_{1}, n_{1}} T_{x_{2}} \tilde{T}_{y_{2}} M_{m_{2}, n_{2}}=\exp \left(-2 \pi i\left(m_{1} x_{2}+n_{1} y_{2}+\alpha x_{2} y_{1}\right)\right) T_{x_{1}+x_{2}} \tilde{T}_{y_{1}+y_{2}} M_{m_{1}+m_{2}, n_{1}+n_{2}}
$$

or, more suggestively,

$$
L_{\left(\mathbf{x}_{1}, \mathrm{~m}_{1}\right)} L_{\left(\mathbf{x}_{2}, \mathrm{~m}_{2}\right)}=\exp \left(-2 \pi i\left(m_{1} x_{2}+n_{1} y_{2}+\alpha x_{2} y_{1}\right)\right) L_{\left(\mathbf{x}_{1}+\mathbf{x}_{2}, \mathrm{~m}_{1}+\mathrm{m}_{2}\right)} .
$$

where $\mathbf{x}_{\imath}=\left(x_{\imath}, y_{\imath}\right), \mathbf{m}_{\imath}=\left(m_{\imath}, n_{\imath}\right)$, and $L_{\left(\mathbf{x}_{1}, \mathrm{~m}_{1}\right)}=T_{x_{1}} W_{y_{1}} M_{m_{1}, n_{1}}$. That is, we have a $\sigma$ representation $L$ of $\mathbb{R}^{2} \times \mathbb{Z}^{2}$ on $L^{2}\left(\mathbb{R}^{2}\right)$, where $\sigma$ is the 2-cocycle determined from (1.2) :

$$
\sigma\left(\left(\mathbf{x}_{1}, \mathbf{m}_{1}\right),\left(\mathbf{x}_{2}, \mathbf{m}_{2}\right)\right)=\exp \left(2 \pi i\left(m_{1} x_{2}+n_{1} y_{2}+\alpha x_{2} y_{1}\right)\right.
$$

The algebra of observables is taken to be the $C^{*}$-algebra generated by the resolvent of the Hamiltonian (1.1) and its translates by $\left\{T_{x}, \tilde{T}_{y} \mid x, y \in \mathbb{R}\right\}$.We will show later that this algebra coincides with the twisted group $C^{*}$-algebra algebra $C^{*}\left(\mathbb{R}^{2} \times \mathbb{Z}^{2}, \sigma\right)$, generated by the integrated form of the $\sigma$-representation $L$. 


\section{$\S$ 2.2. The Discrete Model}

The model, as originally presented [15], is a many body one of electrons hopping on a two dimensional lattice, which is pierced by a (perpendicular) magnetic field. We first introduce the algebra of the canonical anti-commutation relations over $l^{2}\left(\mathbb{Z}^{2}\right)$ generated by

$$
\left\{a_{m, n}^{+} \cdot a_{m, n} \mid(m, n) \in \mathbb{Z}^{2}\right\}
$$

which satisfy

$$
a_{m, n} a_{m^{\prime}, n^{\prime}}+a_{m^{\prime}, n^{\prime}} a_{m, n}=0, \quad a_{m, n} a_{m^{\prime}, n^{\prime}}^{+}+a_{m^{\prime}, n^{\prime}}^{+} a_{m, n}=\delta_{m, m^{\prime}} \delta_{n^{\prime}, n^{*}}
$$

Kohmoto and Fradkin [15] give the Hamiltonian $\mathbf{H}$ for the model as the formal sum:

$$
\sum_{m, n}\left(a_{m+1, n}^{+} a_{m, n}+a_{m-1, n}^{+} a_{m, n}+e^{2 \pi a m} a_{m, n+1}^{+} a_{m, n}+e^{-2 \pi a m} a_{m, n-1}^{+} a_{m, n}\right) .
$$

Again $\alpha$ is just the flux through a unit cell of the lattice and $a_{m, n}^{+}, a_{m, n}$ are creation and annihilation operators for electrons at position $(m, n)$ of the lattice $\mathbb{Z}^{2}$. We recast this in a mathematically simpler form by working on the one particle space (which is possible as the Hamiltonian is only quadratic). Thus letting $\phi=$ $\sum_{m, n} \lambda_{m, n} a_{m, n}^{+}$for $\lambda_{m, n} \in l^{2}\left(\mathbb{Z}^{2}\right)$ and calculating $[\mathbb{H}, \phi]$ we see that $\mathbb{H}$ yields an operator $H$ on $l^{2}\left(\mathbb{Z}^{2}\right)$ with the following action:

$$
\begin{aligned}
(H \lambda)_{m, n} & =\lambda_{m+1, n}+\lambda_{m-1, n}+e^{2 \pi i \alpha m} \lambda_{m, n-1}+e^{-2 \pi i \alpha m} \lambda_{m, n-1} \\
& =\left(\left(U+U^{*}+V+V^{*}\right) \lambda\right)_{m, n},
\end{aligned}
$$

where $U$ shifts the $x$ label by one and $V$ is a twisted shift operator in the $y$ direction. We refer to (1.5) as the Hamiltonian of the discrete system.

The Hall conductance of the system is given in [15] as

$$
\sigma_{H}=\frac{e^{2}}{\hbar} \sum_{E_{\alpha}<E_{F}} \sum_{E_{\beta}>E_{F}} \hbar^{2}\left(\frac{\left(v_{y}\right)_{\alpha \beta}\left(v_{x}\right)_{\beta \alpha}-\left(v_{x}\right)_{\alpha \beta}\left(v_{y}\right)_{\beta \alpha}}{\left(E_{\alpha}-E_{\beta}\right)^{2}}\right),
$$

where $\alpha$ and $\beta$ are indices labelling the states, $E_{\alpha}$ is the energy level of state $\alpha$, and $E_{F}$ is the Fermi level. The explicit forms of the velocity operators $v_{x}$ and $v_{y}$ are 


$$
\begin{aligned}
& v_{x}=\frac{1}{i \hbar} \sum_{m, n}\left(a_{m+1, n}^{+} a_{m, n}-a_{m, n}^{+} a_{m+1, n}\right) \\
& v_{y}=\frac{1}{i \hbar} \sum_{m, n}\left(e^{2 \pi i \alpha m} a_{m, n+1}^{+} a_{m, n}-e^{-2 \pi i \alpha m} a_{m, n}^{+} a_{m, n+1}\right) .
\end{aligned}
$$

Simple calculations show that as operators on $l^{2}\left(\mathbb{Z}^{2}\right)$ they have the form

$$
\begin{gathered}
\left(v_{x} \lambda\right)_{m, n}=\frac{1}{i \hbar}\left(\lambda_{m+1, n}-\lambda_{m-1, n}\right)=\frac{1}{i \hbar}\left(\left(U-U^{*}\right) \lambda\right)_{m, n} \\
\left(v_{y} \lambda\right)_{m, n}=\frac{1}{i \hbar}\left(e^{2 \pi i \alpha m} \lambda_{m, n+1}-e^{-2 \pi i \alpha m} \lambda_{m, n-1}\right)=\frac{1}{i \hbar}\left(\left(V-V^{*}\right) \lambda\right)_{m, n} .
\end{gathered}
$$

We note here that the expression for $\sigma_{H}$ given above is limited to rational values of $\alpha$, and explain below how to define the conductance for any value of the flux $\alpha$. The coincidence of the two definitions for $\alpha=p / q$ is also demonstrated.

Observe now that the unitary operators $U$ and $V$ satisfy $U V=e^{2 \pi i \alpha} V U$. Setting

$$
(\hat{U} \lambda)_{m, n}=e^{-2 \pi i \alpha n} \lambda_{m-1, n} \text { and }(\hat{V} \lambda)_{m, n}=\lambda_{m, n-1}
$$

yields the precise discrete analogues of the magnetic translations, for

$$
[H, \hat{U}]=0=[H, \hat{V}] \text { and } \hat{U} \hat{V}=e^{-2 \pi i \alpha} \hat{V} \hat{U} .
$$

This results from recognising $U$ and $V$ as generators of a right $\gamma$-representation of $\mathbb{Z}^{2}$, and deriving the corresponding left $\bar{\gamma}$-representation, with which it commutes. Here $\gamma$ is the 2 -cocycle on $\mathbb{Z}^{2}$ given by

$$
\gamma((m, n),(\bar{m}, \bar{n}))=\exp (2 \pi i \alpha m \bar{n}) .
$$

So $\hat{U}$ and $\hat{V}$ are symmetries of the system under consideration, and any observables must commute with them. Thus the algebra of observables is contained in $\{\hat{U}, \hat{V}\}^{\prime} \subset \mathscr{B}\left(l^{2}\left(\mathbb{Z}^{2}\right)\right)$. Note that this commutant contains the $C^{*}$-algebra generated by $U$ and $V$, and that the latter contains the Hamiltonian and the velocity operators. It is also closed under the $\mathbb{R}^{2}$ action

$$
(x, y)\left(U^{m} V^{n}\right)=e^{2 \pi i(x m+y n)} U^{m} V^{n}
$$

Letting $\delta_{1}$ and $\delta_{2}$ denote the corresponding derivations,

$$
\delta_{1}\left(U^{m} V^{n}\right)=2 \pi i m U^{m} V^{n} \text { and } \delta_{2}\left(U^{m} V^{n}\right)=2 \pi i n U^{m} V^{n},
$$


the velocity operators may be written

$$
v_{x}=\frac{-1}{h} \delta_{1}(H) \text { and } v_{y}=\frac{-1}{h} \delta_{2}(H)
$$

We choose the $C^{*}$-algebra generated by $U$ and $V$ as the algebra of observables for the discrete system. It is known as the (rational or irrational) rotation algebra according to the rationality or otherwise of the number $\alpha$ in the relation $U V=\exp (2 \pi i \alpha) V U$. The most useful characterization of $A_{\alpha}$ for our purposes is as a twisted group $C^{*}$-algebra. As was noted above, $U$ and $V$ generate a $\gamma$ representation of $\mathbb{Z}^{2}$, and any such representation extends canonically [2] to give first a representation of $L^{1}\left(\mathbb{Z}^{2}, \gamma\right)$, and then, upon taking the $C^{*}$-envelope of this algebra, to $C^{*}\left(\mathbb{Z}^{2}, \gamma\right)$. Of course $\gamma$ is only determined up to a cohomology class, and cohomologous cocycles produce isomorphic algebras. This freedom corresponds exactly to the choice of gauge for the potential that generates the magnetic field.

\section{§ 2.3. Inducing Representations}

We observe in this section that there is a one to one correspondence between primary representations of $C^{*}\left(\mathbb{R}^{2} \times \mathbb{Z}^{2}, \sigma\right)$ and primary representations of $C^{*}\left(\mathbb{Z}^{2}, \omega\right)$, where $\omega$ is a cocycle cohomologous to $\gamma$. For concreteness, and because the analysis is relatively transparent in this gauge, we consider here the cocycle (1.3) on $\&=\mathbb{R}^{2} \times \mathbb{Z}^{2}$ (although we will choose other cocycles later where these simplify the calculation at hand). Let $\mathscr{K}$ denote the subgroup $(0,0) \times \mathbb{Z}^{2} \subset$ ङ5. Then the restriction of the cocycle to $\mathscr{K}$ is trivial, so $\hat{\mathscr{K}}^{\sigma}$, the $\sigma$-dual of $\mathscr{K}$ (that is, the set of equivalence classes of irreducible $\sigma$-representations of $\mathscr{K}$ ) is simply

$$
\hat{\mathscr{K}}^{\sigma}=\hat{\mathscr{K}} \cong \hat{\mathbb{Z}}^{2} \cong \mathbb{T}^{2}
$$

So each irreducible $\sigma$-representation $L_{0}$ of $\mathscr{K}$ is given by a character:

$$
L_{0}(m, n)=\text { multiplication by } \exp (2 \pi i(\theta m+\phi n))
$$

for some $(\theta, \phi) \in \mathbb{T}^{2}$. Here of course $(m, n)$ is identified with $(0,0, \mathrm{~m}, \mathrm{n}) \in \mathscr{K}$.

For any abelian subgroup $\mathscr{C}$ of \&s there is a canonical action of \&s on $\hat{\mathscr{C}}^{\sigma}$ given by $s\left(L_{0}\right)=L_{0}^{s}$, for $L_{0}^{s}$ the $\sigma$-representation of $\mathscr{C}$ defined by

$$
c \mapsto \frac{\sigma\left(s^{-1}, s\right)}{\sigma\left(s c, s^{-1}\right) \sigma(s, c)} L_{0}(c),
$$


where $c \in \mathscr{C}$ and $s \in \mathscr{S}$. $^{1}$

Simple calculations with the above cocycle on the subgroup $\mathscr{K}$ of \& show that if $s=(x, y, m, n)$ and $k=(0,0, \bar{m}, \bar{n})$ then

$$
L_{0}^{s}(k)=\exp (2 \pi i((\theta+x) \bar{m}+(\phi+y) \bar{n})) L_{0}(k)
$$

So the action on the characters is reflecting the $\mathbb{R}^{2}$ action on $\mathbb{T}^{2}$. The representation $L_{0}$ is invariant under the action of $s \in \&$ if we have

$$
\exp (2 \pi i(x \bar{m}+y \bar{n}))=1 \forall(\bar{m}, \bar{n}) \in \mathbb{Z}^{2}
$$

Setting $\bar{m}=0$ gives $x \in \mathbb{Z}$, and setting $\bar{n}=0$ gives $y \in \mathbb{Z}$. Thus the subgroup of \&5 under whose action the representation $L_{0}$ of $\mathscr{K}$ is invariant is simply

$$
\mathscr{G}=\{(k, l, m, n): k, l, m, n \in \mathbb{Z}\}=\mathbb{Z}^{2} \times \mathbb{Z}^{2} \subset \mathbb{R}^{2} \times \mathbb{Z}^{2}
$$

Suppose that $\mathscr{G}$ is a closed subgroup of the separable, locally compact group (S), and that $\sigma$ is a cocycle on \&s. Note that the restriction of $\sigma$ gives a cocycle on $\mathscr{G}$, and let $L$ be a $\sigma$-representation of $\mathscr{G}$ on $\mathscr{H}(L)$. Now assume that there is an invariant measure $\mu$ on $\$ S / \mathscr{G}$, and consider the space of functions $f: \& \rightarrow \mathscr{H}(L)$ which satisfy

1. $(f(x), h)$ is a Borel function of $x \forall h \in \mathscr{H}(L)$.

2. $f(\xi x)=\sigma(\xi, x) L_{\xi}(f(x)) \forall \xi \in \mathscr{G}, x \in$ \&).

3. $\int_{\text {S } / \mathscr{G}}(f(x), f(x)) d u(z)<\infty$.

The $\sigma$-representation of \& induced from $L$ will be denoted $\sigma-L \uparrow \stackrel{\leftrightarrow}{\mathscr{G}}$, and is defined on the Hilbert space defined above by

$$
((\sigma-L \uparrow \underset{\mathscr{G}}{\stackrel{\leftrightarrow}{(S)}}(y)) f)(x)=\frac{1}{\sigma(x, y)} f(x y) .
$$

Note that taking $\mathscr{G}$ to be the subgroup given by the identity in \&s yields the (right) $\sigma$-regular representation of \&s.

Theorem 8.1 of [21] shows that any primary $\sigma$-representation $L$ of \&s for which $\left.L\right|_{\mathscr{K}}$ is a multiple of $L_{0}$ can be induced to give a primary $\sigma$-representation

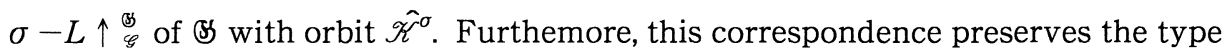
of the representations, and any primary $\sigma$-representation of \&s with the required restriction property will arise from such an $L$. Indeed, because there is a single orbit of $\hat{\mathscr{K}}^{\sigma}$ under \&s, all of the primary $\sigma$-representations of \&s have this form.

\footnotetext{
${ }^{1}$ See the discussion in $[21]$ following Lemma 4.2, and note that \& is abelian here.
} 
Thus the problem of calculating the primary $\sigma$-representations of \& has been reduced to that of finding the primary $\sigma$-representations of $\mathscr{G}$. In what follows it is further reduced to the study of primary $\omega$-representations of $\mathscr{G} / \mathscr{K}$, where the cocycle $\omega$ remains to be defined.

Note firstly the form of the cocycle on the subgroup $\mathscr{G}$ of \&:

$$
\sigma((k, l, m, n),(\bar{k}, \bar{l}, \bar{m}, \bar{n}))=\exp (2 \pi i \alpha l \bar{k}) .
$$

If $f$ denotes the projection of $\mathscr{G}$ onto $\mathscr{G} / \mathscr{K}$ then we obviously have $\sigma=\omega_{0} f$, for $\omega$ a cocycle on $\mathscr{G} / \mathscr{K}$. Clearly the cocycle $\omega$ on $\mathscr{G} / \mathscr{K} \cong \mathbb{Z}^{2}$ is the cocycle $\gamma$ defined in (1.7), so that $C^{*}(\mathscr{G} / \mathscr{K}, \omega) \cong A_{\alpha}$. So in the statement of Theorem 8.2 of [21] we can simply take $\tau \equiv 1$ on $\mathscr{G}$, so that $\tau$-representations of $\mathscr{G}$ are, as for $\mathscr{K}$, simply characters. The restriction property required by the theorem (essentially, that given $L \in \hat{\mathscr{K}}^{\sigma}$ there should be a $\tau$-representation $M$ of $\mathscr{G}$ which restricts to $L$ on $\mathscr{K})$ is therefore satisfied trivially.

Suppose now that we are given an $\omega$-representation $N$ of $\mathscr{G} / \mathscr{K}$ and a representation $L_{0}$ of $\mathscr{K}$. Then letting $N^{f}$ denote the representation of $\mathscr{G}$ given by $\omega_{0} f$ and $M$ a representation of $\mathscr{G}$ as promised above, the map $N \mapsto M \otimes N^{f}$ implements the equivalence between primary $\omega$-representations of $\mathscr{G} / \mathscr{K}$ and primary $\sigma$-representations of $\mathscr{G}$ which reduce to a multiple of $L_{0}$ on $\mathscr{K}$. The type of the representation is preserved under this map, as is the irreducibility of the original representation. We have now established the following result.

Proposition 1. The map

$$
N \mapsto \sigma-\left(M \otimes N^{f}\right) \uparrow \stackrel{\leftrightarrow}{\mathscr{G}}
$$

is one to one between equivalence classes of primary $\omega$-representations of $\mathscr{G} / \mathscr{K} \cong \mathbb{Z}^{2}$ and primary $\sigma$-representations of \&s which have $\mathbb{T}^{2}$ as their orbit. All primary $\sigma$ representations have this restriction property due to the transitivity of $\hat{\mathscr{K}}^{\sigma}$ under the action of \&s.

Thus the claim made at the beginning of this section about the equivalence of the factor representations for the discrete and continuous models is proven. We further note that tracing through the proofs of the various results used above, it is easily established that the following commutants are isomorphic:

$$
N^{\prime} \cong\left(M \otimes N^{f}\right)^{\prime} \cong\left(\sigma-\left(M \otimes N^{f}\right) \uparrow \underset{G}{\stackrel{f}{G}}\right)^{\prime}
$$




\section{§ 3. Relating the Algebras}

\section{§ 3.1. The Rotation Algebras}

Apart from the characterization as a twisted group $C^{*}$-algebra mentioned above, $A_{\alpha}$ is probably best known as the $C^{*}$-algebra associated with the dynamical system $\left(\mathbb{S}^{1}, \mathbb{Z}, \varrho\right)$, for $\varrho$ the homeomorphism of $\mathbb{S}^{1}$ given by rotation through an angle of $2 \pi \alpha$. That is, $A_{\alpha} \cong C\left(\mathbb{S}^{1}\right) \times \varrho \mathbb{Z}$, where $\varrho$ is also used to denote the corresponding action on $C\left(\mathbb{S}^{1}\right)$ :

$$
(\varrho f)\left(e^{2 \pi i r}\right)=f\left(e^{2 \pi(r-\alpha)}\right) .
$$

While the irrational rotation algebras are known to possess a unique normalised trace and to be simple, the same is certainly not true of the rational rotation algebras. One of the many surprising properties of the rotation algebras is that they are all essentially non-isomorphic: more precisely,

$$
A_{\alpha} \cong A_{\alpha^{\prime}} \Leftrightarrow \alpha= \pm \alpha^{\prime} \bmod \mathbb{Z}
$$

so that the only $*$-isomorphisms are the obvious ones between $A_{\alpha}$ and $A_{\alpha+n}$ for $n \in \mathbb{Z}$ (see the defining relation), and that between $A_{\alpha}$ and $A_{-\alpha}$ given by exchanging $V$ and $U$. See [18] and [20] for more details.

\section{§ 3.2. Strong Morita Equivalence of $C^{*}$-algebras}

A somewhat weaker notion of equivalence than isomorphism sheds more light on the relationship between the various rotation algebras. Rieffel defines two $C^{*}$-algebras $A$ and $B$ to be strongly Morita equivalent (SME) if there is an "imprimitivity bimodule", or " $A-B$ equivalence bimodule" for the two algebras [28]. For separable $C^{*}$-algebras strong Morita equivalence is equivalent to stable isomorphism. That is, $A$ is SME to $B$ if and only if $A \otimes \mathscr{K} \cong B \otimes \mathscr{K}$ for $\mathscr{K}$ the compact operators on a separable Hilbert space. Rieffel's paper [29] contains the following fact:

Theorem 1. Let $G$ be a locally compact group, and H,K locally compact subgroups of $G$. Let $K$ act on the left on $G / H$ and let $C^{*}(K, G / H)$ denote the corresponding transformation group $C^{*}$-algebra. Similarly, let $C^{*}(H, K \backslash G)$ denote the transformation group $C^{*}$-algebra for the action of $H$ on the right on $K \backslash G$. Then $C^{*}(K, G / H)$ is strongly Morita equivalent to $C^{*}(H, K \backslash G)$. 
Consider then $G=\mathbb{R}^{2}, K=\mathbb{Z}^{2}$ and $H=\{(v, \alpha v) ; v \in \mathbb{R}\}=\mathbb{R}(1, \alpha)$ and apply the theorem. It's clear that $H$ acting on $G / K$ (the left/right distinction not arising for the abelian groups considered here) is the Kronecker foliation of the torus of slope $\alpha$. Consider then $K$ acting on $\mathbb{R}^{2} / H$ :

$$
\begin{aligned}
(m, n)((a, b)+\mathbb{R}(1, \alpha)) & =(m+a, n+b)+\mathbb{R}(1, \alpha) \\
& =(a, b+(n-\alpha m))+\mathbb{R}(1, \alpha) .
\end{aligned}
$$

This gives a $\mathbb{Z}^{2}$ action on $\mathbb{R}$,

$$
(m, n)[x]=[x+(n-\alpha m)],
$$

the corresponding algebra of which is known to be isomorphic to that of the Kronecker flow of slope $-1 / \alpha$. This can be seen [7] by Fourier transforming the usual representation of the transformation group $C^{*}$-algebra $C_{0}(\mathbb{R}) \times{ }_{a} \mathbb{Z}^{2}$ on $L^{2}\left(\mathbb{R}^{2} \times \mathbb{Z}^{2}\right)$, and noting that the result is a representation of $C\left(\mathbb{T}^{2}\right) \times{ }_{\beta} \mathbb{R}$ for the appropriate action $\beta$ of $\mathbb{R}$ on $\mathbb{T}^{2}$. So if $\mathscr{F}$ denotes the $C^{*}$-algebra of the Kronecker flow we have

$$
\mathscr{F}_{\alpha} \mathrm{SME} \mathscr{F}_{-1 / \alpha} .
$$

Now consider the situation in which $G=\mathbb{S}^{1} \times \mathbb{R}, K=\{(v, \alpha v): v \in \mathbb{R}\}$ where the first component is always taken modulo $\mathbb{Z}$, and $H=\mathbb{Z} \times\{0\}$. Then again (by construction) the algebra $C^{*}(K, G / H)$ is simply $\mathscr{F}_{\alpha}$, and we need to examine the action of $H$ on $G / K=\mathbb{S}^{1} \times \mathbb{R} / \sim$, where

$$
(\theta, x) \sim(\phi, y) \text { if } \exists b \in \mathbb{R} \text { with }(\theta+b, x+\alpha b)=(\phi, y)
$$

Now if $h \in H$ we have

$$
h(\theta, x)=(\theta, x+h) \sim\left(\theta-\frac{x+h}{\alpha}, 0\right) .
$$

That is, the action is equivalent to that of $\mathbb{Z}$ on $\mathbb{S}^{1}$ by the irrational rotation,

$$
h(\theta)=\left(\theta-\frac{h}{\alpha}\right)
$$

and so the second Morita equivalence is established:

$$
\mathscr{F}_{\alpha} \mathrm{SME} A_{-1 / \alpha}
$$

Thus in combination we have 


$$
\mathscr{F}_{\alpha} \operatorname{SME} \mathscr{F}_{-1 / \alpha} . \operatorname{SME} A_{\alpha}
$$

From these results it follows easily that $A_{\alpha}$ is strong Morita equivalent to $A_{\beta}$ if $\alpha=B \beta$, where $B \in G L_{2}(\mathbb{Z})$ acts on $\beta$ by

$$
\left[\begin{array}{ll}
a & b \\
c & d
\end{array}\right] \beta=\frac{a \beta+b}{c \beta+d} .
$$

This somewhat mysterious behaviour is explained [26] by considering the corresponding dynamical systems. The Kronecker flow on the torus is known to arise by taking the flow under the constant function for the dynamical system $\left(\mathbb{S}^{1}, \mathbb{Z}, \alpha\right)$. We briefly recall the definition of the latter, and demonstrate the proposed relationship. The flow under the constant function in this case is defined on the space $X=\mathbb{R} \times \mathbb{S}^{1} / \sim$, where $(r,[s]) \sim\left(r^{\prime},\left[s^{\prime}\right]\right)$ if there is an $m \in \mathbb{Z}$ with $r=r^{\prime}+m$ and $[s]=\left[s^{\prime}+m \alpha\right]$. Here $[s]$ denotes the class of $s \in \mathbb{R}$ in $\mathbb{R} / \mathbb{Z}$. We use $[r,[s]]$ to label the class of $(r,[s])$ in $X$. Note that the space $X$ is homeomorphic to a torus: the map $\pi: X \rightarrow \mathbb{T}^{2}$ given by

$$
\pi([r,[s]])=([r],[s+\phi r])
$$

is continuous and has a continuous inverse

$$
\left.\pi^{-1}([r],[s]]\right)=[r,[s-\phi r]]
$$

Easy calculations show that these maps are well defined with respect to the equivalence relation. The flow $\phi$ under the constant function is defined by

$$
\phi(t)[r,[s]]=[r+t,[s]],
$$

and we note that

$$
\pi_{0} \phi(t)[r,[s]]=([r+t],[s+\phi r+\phi t])=k(t)([r],[s+\phi r])=k(t)_{0} \pi[r,[s]],
$$

where $k$ denotes the Kronecker flow on the torus, defined by

$$
k(t)([r],[s])=([r+t],[s+\phi t]) .
$$

So the flow under the constant function for the $\mathbb{Z}$-dynamical system defined above is indeed flow-equivalent to the Kronecker flow. 
Further results of Green [17] that we discuss later imply that the $C^{*}$-algebra $\mathscr{F}_{\alpha}$ is stable, and thus we know that $A_{\alpha} \otimes \mathscr{K} \cong \mathscr{F}_{\alpha}$. So strong Morita equivalence of $A_{\alpha}$ and $A_{\beta}$ for $\alpha=B \beta$ corresponds to isomorphism of the flow algebras $\mathscr{F}_{\alpha}$ and $\mathscr{F}_{\beta}$. But the dynamical systems here are flow equivalent, corresponding to the homeomorphism of the torus defined by $B$, so the isomorphism between the algebras is clear. Of course this works more generally: if systems given by taking the flow under the constant function of the two dynamical systems $A=(C(X), \mathbb{Z}, \alpha)$ and $B=(C(X), \mathbb{Z}, \beta)$ are flow equivalent then the $C^{*}$-algebras of $A$ and $B$ are strongly Morita equivalent (see [22]).

The relevance of the above discussion to the physical models under consideration will soon become clear, in particular with the realization of the isomorphism between the algebra of observables in the continuum case and $\mathscr{F}_{\alpha}$. In summary, the dynamical system underpinning the continuum model is the $\mathbb{R}$-dynamical system obtained by taking the flow under the constant function of the $\mathbb{Z}$-dynamical system of the discrete model.

\section{§ 3.3. Representation Theory for the Continuous Model}

There are a number of equivalent ways of presenting the representations of the algebra of observables for the continuous model $C^{*}\left(\mathbb{R}^{2} \times \mathbb{Z}^{2}, \sigma\right)$ and we need several of them to compare our point of view with that of [34]. Consider for example the cocycle defined by (1.3). Note firstly that if $\rho\left(\mathbf{x}_{\mathbf{i}}, \mathbf{m}_{\mathbf{i}}\right)=$ $\exp \left(-2 \pi i \alpha x_{i} y_{i}\right)$ then

$$
\frac{\rho\left(\mathbf{x}_{1}+\mathbf{x}_{2}, \mathbf{m}_{1}+\mathbf{m}_{2}\right)}{\rho\left(\mathbf{x}_{1}, \mathbf{m}_{1}\right) \rho\left(\mathbf{x}_{2}, \mathbf{m}_{2}\right)} \sigma\left(\left(\mathbf{x}_{1}, \mathbf{m}_{1}\right),\left(\mathbf{x}_{2}, \mathbf{m}_{2}\right)\right)=\sigma_{1}\left(\left(\mathbf{x}_{1}, \mathbf{m}_{1}\right),\left(\mathbf{x}_{2}, \mathbf{m}_{2}\right)\right)
$$

where the new cocycle $\sigma_{1}$ is given by

$$
\sigma_{1}\left(\left(\mathbf{x}_{1}, \mathbf{m}_{1}\right),\left(\mathbf{x}_{2}, \mathbf{m}_{2}\right)\right)=\exp \left(2 \pi i\left(m_{1} x_{2}+n_{1} y_{2}-\alpha x_{1} y_{2}\right)\right)
$$

So $\sigma$ and $\sigma_{1}$ are cohomologous, and a similar calculation with $\rho^{\prime}\left(\mathbf{x}_{\mathbf{i}}, \mathbf{m}_{\mathbf{i}}\right)$ $=\exp \left(-2 \pi i\left(m_{i} x_{i}+n_{i} y_{i}\right)\right)$ shows that $\sigma$ and $\sigma_{1}$ are cohomologous to $\sigma_{2}$ defined by

$$
\sigma_{2}\left(\left(\mathbf{x}_{1}, \mathbf{m}_{1}\right),\left(\mathbf{x}_{2}, \mathbf{m}_{2}\right)\right)=\exp \left(2 \pi i\left(-m_{2} x_{1}+n_{2} y_{1}-\alpha y_{2} x_{1}\right)\right)
$$

Concentrating first on $\sigma_{1}$ above, the $\sigma_{1}$ regular representation $\pi_{1}$ is given by

$$
\left(\pi_{1}\left(\mathbf{x}_{1}, \mathbf{m}_{1}\right) f\right)\left(\mathbf{x}_{2}, \mathbf{m}_{2}\right)=\sigma_{1}\left(\left(\mathbf{x}_{2}, \mathbf{m}_{2}\right),\left(\mathbf{x}_{1}, \mathbf{m}_{1}\right)\right)^{-1} f\left(\mathbf{x}_{1}+\mathbf{x}_{2}, \mathbf{m}_{1}+\mathbf{m}_{2}\right),
$$


where $f \in L^{2}\left(\mathbb{R}^{2} \times \mathbb{Z}^{2}\right)$. After Fourier transforming on the $\mathbb{Z}^{2}$ components, by defining

the representation reads

$$
\hat{f}\left(\mathbf{x}_{2}, \theta, \phi\right)=\sum_{m_{2} n_{2}} \exp \left(-2 \pi i\left(m_{2} \theta+n_{2} \phi\right)\right) f\left(\mathbf{x}_{2}, \mathbf{m}_{2}\right),
$$

$$
\begin{aligned}
& \left(\pi_{1}\left(\widehat{\mathbf{x}_{1}, \mathbf{m}_{1}}\right) f\right)\left(\mathbf{x}_{2}, \theta, \phi\right) \\
& =\exp \left(2 \pi i\left(m_{1}\left(\theta+x_{1}\right)+n_{1}\left(\phi+y_{1}\right)+\alpha y_{1} x_{2}\right)\right) \hat{f}\left(\mathbf{x}_{1}+\mathbf{x}_{2}, \theta+x_{1}, \phi+y_{1}\right) .
\end{aligned}
$$

This representation of the algebra of observables is recognisable as a covariant representation of the twisted dynamical system $\left(C\left(\mathbb{T}^{2}\right), \mathbb{R}^{2}, \beta, u\right)$, for $(\beta, u)$ the appropriate twisted action [24]: $\beta(\mathbf{x})$ is simply translation by $\mathbf{x}$ in the torus variables on $\mathrm{C}\left(\mathbb{T}^{2}\right)$, and $u(\mathbf{x}, \mathbf{y})$ is multiplication by $\exp \left(2 \pi i y_{1} x_{2}\right)$. This may be seen most easily by considering the integrated form. If $g$ is in $L^{1}\left(\mathbb{R}^{2}, C\left(\mathbb{T}^{2}\right), \beta, u\right)$ then the integrated form of (2.1) reads

$$
\left(\tilde{\pi}_{1}(g) f\right)\left(\mathbf{x}_{1}, \theta, \phi\right)=\int_{\mathbb{R}^{2}} d \mathbf{x}_{2}\left(g\left(\mathbf{x}_{2}\right)\right)(\theta, \phi) \exp \left(2 \pi i \alpha x_{1} y_{2}\right) f\left(\mathbf{x}_{1}+\mathbf{x}_{2}, \theta+x_{2}, \phi+y_{2}\right),
$$

which is precisely the representation detailed in equation 1.6 of [34], modulo the sign of the exponential. ${ }^{2}$ Following [34] we denote by $C^{*}\left(\mathbb{T}^{2}, \mathbb{R}^{2}, \beta\right)$ the $C^{*}$-algebra generated by the operators $\tilde{\pi}_{1}(g)$.

Choosing $\sigma_{2}$ for the cocycle and calculating as for $\sigma_{1}$ yields

$$
\left(\pi_{2}\left(\widehat{\mathbf{x}}_{1}, \mathbf{m}_{1}\right) f\right)\left(\mathbf{x}_{2}, y_{2}, \theta, \phi\right)=\exp \left(2 \pi i\left(m_{1}\left(\theta+x_{2}\right)+n_{1}\left(\phi+y_{2}\right)+\alpha y_{1} x_{2}\right)\right) \hat{f}\left(\mathbf{x}_{1}+\mathbf{x}_{2}, \theta, \phi\right)
$$

Notice that for any $(\theta, \phi) \in \mathbb{T}^{2}$ this expression defines a $\sigma_{2}$-representation of $\mathbb{R}^{2} \times \mathbb{Z}^{2}$ on $L^{2}\left(\mathbb{R}^{2}\right)$. So the regular $\sigma_{2}$-representation is decomposable with respect to the direct integral decomposition:

$$
L^{2}\left(\mathbb{R}^{2} \times \mathbb{T}^{2}\right)=\int_{\mathbb{T}^{2}}^{\oplus} L_{\theta, \phi}^{2}\left(\mathbb{R}^{2}\right) d u(\theta, \phi)
$$

Writing $\omega$ for $(\theta, \phi)$ and $f_{\omega}\left(x_{1}, y_{1}\right)$ for $\hat{f}\left(x_{1}, y_{1}, \theta, \phi\right)$ we obtain $\sigma_{2}$ representations of $\mathbb{Z}^{2}$ and $\mathbb{R}^{2}$ on $L_{\omega}^{2}\left(\mathbb{R}^{2}\right)$ via

$$
\begin{aligned}
\left(\pi_{2}\left(m_{1}, n_{1}\right) f_{\omega}\right)\left(x_{2}, y_{2}\right) & =\exp \left(2 \pi i\left(m_{1}\left(\theta+x_{2}\right)+n_{1}\left(\phi+y_{2}\right)\right)\right) f_{\omega}\left(x_{2}, y_{2}\right) \\
\left(\pi_{2}\left(x_{1}, y_{1}\right) f_{\omega}\right)\left(x_{2}, y_{2}\right) & =\exp \left(2 \pi i \alpha y_{1} x_{2}\right) f_{\omega}\left(x_{1}+x_{2}, y_{1}+y_{2}\right) .
\end{aligned}
$$

${ }^{2}$ Xia writes " $\beta$ " $=e|B| / h c$, which presumes a $\mathbf{B} \rightarrow-\mathbf{B}$ symmetry that is not evident until later in this paper. 
The integrated form of these representations is

$$
\left(\tilde{\pi}_{2}(g) f_{\omega}\right)\left(\mathbf{x}_{1}\right)=\int_{\mathbb{R}^{2}} d \mathbf{x}_{2}\left(g\left(\mathbf{x}_{2}\right)\right)\left(\theta+x_{1}, \phi+y_{1}\right) \exp \left(2 \pi i \alpha x_{1} y_{2}\right) f_{\omega}\left(\mathbf{x}_{1}+\mathbf{x}_{2}\right),
$$

again in accord with those presented in [34], modulo the cocycle definition mentioned previously. We define $C_{\omega}^{*}\left(\mathbb{T}^{2}, \mathbb{R}^{2}, \beta\right)$ to be the $C^{*}$-algebra generated by the operators $\tilde{\pi}_{2}(g)$ (for a fixed $\omega$ ). Hence the two integrated representations of interest, one decomposable and the other not, are simply regular representations for two cohomologous forms of $\sigma$. As such they're obviously unitarily equivalent, and an easy calculation demonstrates that if $g \in L^{1}\left(\mathbb{R}^{2}, C\left(\mathbb{T}^{2}\right), \beta, u\right)$ and $(U f)(x, y, \theta, \phi)=$ $f(x, y, \theta+x, \phi+y)$, for $f \in L^{2}\left(\mathbb{R}^{2} \times \mathbb{T}^{2}\right)$, then $U \tilde{\pi}_{1}(g)=\tilde{\pi}_{2}(g) U$.

\section{§ 3.4. Isomorphisms of Algebras}

In order to demonstrate some of the promised isomorphisms it's necessary to briefly introduce another class of $C^{*}$-algebras, the twisted covariance algebras developed by Green in [16] .

Definition 1. A twisted covariant system $(G, A, \tau)$ consists of a locally compact group $G$, a $C^{*}$ algebra $A$, a strongly continuous left action $(s, a) \mapsto s(a)$ of $G$ by *automorphisms of $A$, and (the "twist") a continuous homomorphism $\tau$ of a closed normal subgroup $N_{\tau}$ of $G$ into the group of unitaries of the multiplier algebra of $A$ (equipped with the strong topology) that satisfies

$$
\tau(n) a \tau\left(n^{-1}\right)=n(a) \text { and } \tau\left(s n s^{-1}\right)=s(\tau(n)) \forall n \in N_{\tau} a \in A, s \in G \text {. }
$$

The twisted covariance algebra corresponding to the above system is a quotient of $C^{*}(G, A)$, for $(G, A)$ the obvious untwisted covariant system contained in the definition. A covariant representation $(U, \pi)$ is said to "preserve $\tau$ " if

$$
U(n)=\pi(\tau(n)) \forall n \in N_{\tau}
$$

Let $\mathscr{M}$ denote the set of $\tau$-preserving covariant representations of $(G, A)$. Then if $I_{\tau}$ is the closed two-sided ideal given by

$$
I_{\tau}=\bigcap_{(U, \pi) \in \mathscr{M}} \operatorname{ker}(U \times \pi),
$$

the twisted covariance algebra $C^{*}(G, A, \tau)$ is just $C^{*}(G, A) / I_{\tau}$. We refer to $[16]$ for the proof that this construction is well defined.

Now apply this to $C^{*}\left(\mathbb{Z}^{2}, \gamma\right)$. Let $\mathbb{Z}^{2} \gamma$ be 


$$
\left\{(m, n, \theta) ;(\mathrm{m}, \mathrm{n}) \in \mathbb{Z}^{2}, \theta \in \mathbb{T}^{1}\right\}
$$

with multiplication defined by

$$
\left(m_{1}, n_{1}, \theta_{1}\right)\left(m_{2}, n_{2}, \theta_{2}\right)=\left(m_{1}+m_{2}, n_{1}+n_{2}, \gamma\left(\left(m_{1}, n_{1}\right),\left(m_{2}, n_{2}\right)\right) \theta_{1} \theta_{2}\right) .
$$

If we define $N_{\tau}$ to be $(0,0) \times \mathbb{T} \subset \mathbb{Z}^{2} \gamma$, and take $N_{\tau} \rightarrow U(\mathbb{C})$ to be the map

$$
\tau(0,0, \theta) z=\theta z
$$

then it's easy to check that we obtain a twisted covariance system $\left(\mathbb{Z}^{2} \gamma, \mathbb{C}, \tau\right)$ if $\mathbb{Z}^{2} \gamma$ acts trivially on $\mathbb{C}$. Now note that $c:(m, n) \mapsto(m, n, 1)$ is a Borel section of $\mathbb{Z}^{2} \gamma, / \mathbb{T} \rightarrow \mathbb{Z}^{2} \gamma ;$ a simple calculation shows

$$
\tau\left(c(m, n) c(\bar{m}, \bar{n}) c(m+\bar{m}, n+\bar{n})^{-1}\right)=\tau(0,0, \gamma((m, n),(\bar{m}, \bar{n}))),
$$

which is simply multiplication by $\gamma((m, n),(\bar{m}, \bar{n}))$.

We recall that $C^{*}\left(\mathbb{Z}^{2} \gamma, \mathbb{C}, \tau\right)$ has $C_{C}\left(\mathbb{Z}^{2} \gamma, \mathbb{C}, \tau\right)$ as a dense $*$-subalgebra, where the latter is

$$
\left\{f: \mathbb{Z}^{2} \gamma \rightarrow \mathbb{C} ; f((0,0, \theta)(m, n, \phi))=f(m, n, \phi) \tau(0,0, \theta)^{-1}=\bar{\theta} f(m, n, \phi)\right\},
$$

in which the image of the support of $f$ in $\mathbb{Z}^{2} \gamma / \mathbb{T}$ is required to be compact [16, page 197]. Given this, Proposition A.1 of Appendix 1 of [23] demonstrates that there is a map $\left.f \mapsto f\right|_{\mathbb{Z}^{2} \times 1}$ from $C_{c}\left(\mathbb{Z}^{2} \gamma, \mathbb{C}, \tau\right)$ to $L^{1}\left(\mathbb{Z}^{2} \gamma / \mathbb{T}\right)$ which extends to give the isomorphism

$$
C^{*}\left(\mathbb{Z}^{2} \gamma, \mathbb{C}, \tau\right) \cong C^{*}\left(\mathbb{Z}^{2} \gamma / \mathbb{T}, \gamma\right)
$$

Given that the latter algebra is isomorphic to $C^{*}\left(\mathbb{Z}^{2}, \gamma\right)$, we've opened up the possibility of applying the results of Green to the algebras of interest in the quantum Hall effect. Of particular moment is his generalized version of Mackey's imprimitivity theorem, which we now use to establish a relationship between the algebras corresponding to the continuous and discrete models.

Proposition 2. $A_{\alpha} \otimes \mathscr{K}\left(L^{2}\left(\mathbb{T}^{2}\right)\right) \cong C\left(\mathbb{T}^{2}\right) \times_{\beta, u} \mathbb{R}^{2}$, where $\beta: \mathbb{R}^{2} \rightarrow \operatorname{Aut}\left(C\left(\mathbb{T}^{2}\right)\right)$ is given by left translation and $u((x, y),(\bar{x}, \bar{y}))$ is multiplication by the following lift of the symmetric cocycle on $\mathbb{Z}^{2}$ to $\mathbb{R}^{2}$ :

\footnotetext{
${ }^{3}$ That is, the cocycle $\bar{\gamma}((m, n),(\bar{m}, \bar{n}))=\exp (\pi i \alpha(m \bar{n}-\bar{m} n))$, which is cohomologous to the cocycle $\gamma$ defined by (1.7).
} 


$$
\bar{\gamma}((x, y),(\bar{x}, \bar{y}))=\exp (\pi i \alpha(x \bar{y}-y \bar{x})) .
$$

Proof. For the groups outlined in the proposition the imprimitivity theorem, Corollary 2.12 of [17], reads

$$
C^{*}\left(\mathbb{Z}^{2} \tilde{\gamma}, \mathbb{C}, \tau\right) \otimes \mathscr{K}\left(L^{2}\left(\mathbb{R}^{2} \tilde{\gamma} / \mathbb{Z}^{2} \tilde{\gamma}\right)\right) \cong C^{*}\left(\mathbb{R}^{2} \tilde{\gamma}, C_{\infty}\left(\mathbb{R}^{2} \tilde{\gamma} / \mathbb{Z}^{2} \tilde{\gamma}\right) \otimes \mathbb{C}, \hat{\tau}\right),
$$

where the twist $\hat{\tau}(n)=\tau(n) \otimes 1$ and $N_{\hat{\tau}}=N_{\tau} \subset \mathbb{Z}^{2} \tilde{\gamma} \subset \mathbb{R}^{2} \tilde{\gamma}$.

Given the above considerations the left hand side here is isomorphic to $A_{\alpha} \otimes \mathscr{K}\left(L^{2}\left(\mathbb{T}^{2}\right)\right)$, so consider the covariance algebra given on the right. As with any such algebra it can be rewritten as a twisted crossed product via the correspondence outlined in [24]. Indeed if $N$ is the subgroup on which the twist is defined then

$$
C^{*}(G, A, \tau) \cong A \times_{\beta, u} G / N,
$$

for the twisted action $(\beta, u)$ defined in [24]. For the algebras at hand, take first the Borel cross section

$$
c: \mathbb{R}^{2} \tilde{\gamma} / N \rightarrow \mathbb{R}^{2} \tilde{\gamma} \text { given by } c(s)=c(s, 1)
$$

Then $\beta_{s N}=\alpha_{c(s)}$. But the action of $\alpha_{c(s)}$ is as follows:

$$
\begin{aligned}
(\alpha(x, y, \theta) f)\left((r, s, \phi)+\mathbb{Z}^{2} \tilde{\gamma}\right) & =f\left((x, y, \theta)+\mathbb{Z}^{2} \tilde{\gamma}\right) \\
& =f\left((x+r, y+s, \theta \phi \tilde{\gamma}((x, y),(r, s)))+\mathbb{Z}^{2} \tilde{\gamma}\right) \\
& =(P f)(x+r, y+s) .
\end{aligned}
$$

Here $P: C_{\infty}\left(\mathbb{R}^{2} \tilde{\gamma} / \mathbb{Z}^{2} \tilde{\gamma}\right) \rightarrow \mathrm{C}\left(\mathbb{T}^{2}\right)$ is given by

$$
(P f)(\{r\},\{s\})=f\left((r, s, \theta)+\mathbb{Z}^{2} \tilde{\gamma}\right),
$$

for $\{a\}$ the fractional part of $a$.

Now, exactly as in the case of $C^{*}\left(\mathbb{Z}^{2}, \tilde{\gamma}\right)$, we have

That is,

$$
u(s N, t N)=\tau\left(c(s) c(t) c(s t)^{-1}\right)=\text { multiplication by } \tilde{\gamma}(s, t)
$$

$$
\begin{aligned}
C^{*}\left(\mathbb{R}^{2} \tilde{\gamma}, \mathrm{C}_{\infty}\left(\mathbb{R}^{2} \tilde{\gamma} / \mathbb{Z}^{2} \tilde{\gamma}\right) \otimes \mathbb{C}, \hat{\tau}\right) & \cong C\left(\mathbb{T}^{2}\right) \times_{\beta, u} \mathbb{R}^{2} \tilde{\gamma} / \mathbb{T} \\
& \cong C\left(\mathbb{T}^{2}\right) \times_{\beta, u} \mathbb{R}^{2},
\end{aligned}
$$

for $\beta$ left translation and $\mu$ defined above. 
We now establish the relevance of this result to the discussion of the continuous model by showing that the algebra $C\left(\mathbb{T}^{2}\right) \times{ }_{\beta, u} \mathbb{R}^{2}$ defined in the previous proposition is isomorphic to the twisted group $C^{*}$-algebra found in $\S 2$.

Proposition 3. Let $\sigma_{3}$ be the cocycle on $\mathbb{R}^{2} \times \mathbb{Z}^{2}$ obtained using the symmetric gauge for the magnetic field:

$$
\sigma_{3}\left(\left(\mathbf{x}_{1}, \mathbf{m}_{1}\right),\left(\mathbf{x}_{2}, \mathbf{m}_{2}\right)\right)=\exp \left(\pi i \alpha\left(x_{1} y_{2}-x_{2} y_{1}\right)\right) \exp \left(2 \pi i\left(m_{1} x_{2}+n_{1} y_{2}\right)\right)
$$

Then $\sigma_{3}$ is cohomologous to $\sigma$, so that $C^{*}\left(\mathbb{R}^{2} \times \mathbb{Z}^{2}, \sigma_{3}\right) \cong C^{*}\left(\mathbb{R}^{2} \times \mathbb{Z}^{2}, \sigma\right)$, and $C^{*}\left(\mathbb{Z}^{2} \times \mathbb{R}^{2}\right.$, $\sigma) \cong C\left(\mathbb{T}^{2}\right) \times_{\beta, u} \mathbb{R}^{2}$

Proof. The first claim is easily established using calculations similar to those contained in $\S 2.3$. Consider then $N=\mathbb{Z}^{2} \times 0$, for which $\left.\sigma_{3}\right|_{N \times N} \equiv 1$. We already have established that

$$
C^{*}\left(G, \sigma_{3}\right) \cong C^{*}\left(G \sigma_{3}, \mathbb{C}, \tau\right),
$$

and Proposition 1.1 of [23] gives

$$
C^{*}\left(G \sigma_{3}, \mathbb{C}, \tau\right) \cong C^{*}\left(G \sigma_{3}, C^{*}\left(N, \operatorname{Res} \sigma_{3}\right), \tau\right),
$$

where $\operatorname{Res} \sigma_{3}$ is the restriction of $\sigma_{3}$ to $N$, and $G \sigma_{3}$ acts on $C^{*}\left(0 \times \mathbb{Z}^{2}\right)$ by

$$
\begin{aligned}
(\beta((x, y, m, n), \theta) f)(0,0, \bar{m}, \bar{n}) & =\bar{\sigma}_{3}((0,0, \bar{m}, \bar{n}),(-x,-y,-m,-n)) f(0,0, \bar{m}, \bar{n}) \\
& =\exp (2 \pi i(x \bar{m}+y \bar{n})) f(0,0, \bar{m}, \bar{n}) .
\end{aligned}
$$

Fourier transforming shows that the action on $C\left(\mathbb{T}^{2}\right)$ is simply translation. The $\tau$-action on $L^{1}\left(\mathbb{Z}^{2}\right)$ is given by

$$
(\tau((0,0, \bar{m}, \bar{n}), \theta) f)(m, n)=\theta f(m-\bar{m}, n-\bar{n}) .
$$

Now we have

$$
C^{*}\left(G \sigma_{3}, C^{*}(N), \tau\right) \cong C^{*}(N) \times_{\beta, u}\left(G \sigma_{3} / N \sigma_{3}\right),
$$

where the $\beta$ action is

$$
(\beta(x, y) \hat{f})(\theta, \phi)=\hat{f}(\theta+x, \phi+y) \text { for } \hat{f} \in C\left(\mathbb{T}^{2}\right)
$$


If we identify $s N$ with $(x, y) \in \mathbb{R}^{2}$ and define the usual cross section $c(s)=(0,0, x, y, 1)$, then a straightforward calculation shows that

$$
u((x, y),(\bar{x}, \bar{y}))=\text { multiplication by } \sigma_{3}((x, y),(\bar{x}, \bar{y}))
$$

That is,

$$
C^{*}\left(G \sigma_{3}, C^{*}(N), \tau\right) \cong C\left(\mathbb{T}^{2}\right) \times_{\beta, u} \mathbb{R}^{2}
$$

This establishes the result.

So, summarizing the sequence of isomorphisms that we've established here,

$$
\begin{aligned}
C^{*}\left(\mathbb{R}^{2} \times \mathbb{Z}^{2}, \sigma\right) \cong C\left(\mathbb{T}^{2}\right) \times_{\beta, u} \mathbb{R}^{2} \cong C^{*}\left(\mathbb{Z}^{2}, \gamma\right) \otimes \mathscr{K}\left(L^{2}\left(\mathbb{T}^{2}\right)\right) \\
=A_{\alpha} \otimes \mathscr{K}\left(L^{2}\left(\mathbb{T}^{2}\right)\right)
\end{aligned}
$$

As mentioned earlier, the $C^{*}$-algebras corresponding to a $\mathbb{Z}$-dynamical system and its $\mathbb{R}$-counterpart formed by taking the flow under the constant function are strong Morita equivalent. For the case of the irrational rotation algebra and its corresponding (Kronecker) flow we show how this can be established by using another result of Green [17]. Let $\mathbb{R}$ act on $C\left(\mathbb{S}^{1}\right) \cong C(\mathbb{R} / \mathbb{Z})$ by

$$
x f(y+\mathbb{Z})=f(y+\alpha x+\mathbb{Z}),
$$

for $f \in C(\mathbb{R} / \mathbb{Z}), x \in \mathbb{R}$. Restricting this action to $\mathbb{Z} \subset \mathbb{R}$ we gain a dynamical system whose $C^{*}$-algebra $C^{*}\left(\mathbb{Z}, C\left(\mathbb{S}^{1}\right)\right)$ is isomorphic to $A_{\alpha}$. Now let $\mathbb{R}$ act on $C\left(\mathbb{S}^{1}\right) \otimes$ $C(\mathbb{R} / \mathbb{Z}) \cong C\left(\mathbb{T}^{2}\right)$ via the diagonal action:

$$
x f_{1}\left(y_{1}+\mathbb{Z}\right) \otimes f_{2}\left(y_{2}+\mathbb{Z}\right)=f_{1}\left(y_{1}+\alpha x+\mathbb{Z}\right) \otimes f_{2}\left(y_{2}+x+\mathbb{Z}\right),
$$

which on $C\left(\mathbb{T}^{2}\right)$ reads

$$
x f\left(\theta_{1}, \theta_{2}\right)=f\left(\theta_{1}+\alpha x, \theta_{2}+x\right) .
$$

Green's result [17] gives the isomorphism

$$
C^{*}\left(\mathbb{R}, C\left(\mathbb{T}^{2}\right)\right) \cong C^{*}\left(\mathbb{Z}, C\left(\mathbb{S}^{1}\right)\right) \otimes \mathscr{K}\left(L^{2}(\mathbb{T})\right) .
$$

The left hand side is of course the Kronecker foliation algebra, with slope $1 / \alpha$. So we have 


$$
\mathscr{F}_{1 / \alpha} \cong A_{\alpha} \otimes \mathscr{K}\left(L^{2}(\mathbb{T})\right)
$$

a result which at first sight merely repeats the result obtained via Morita equivalence, but in fact demonstrates the stability of the $C^{*}$-algebra of the Kronecker flow, as promised earlier. Thus the algebras resulting from the flows on the torus which were described earlier as "strongly Morita equivalent" are in fact isomorphic.

So we now have the promised demonstration that the dynamical system that underlies the continuum model of the quantum Hall effect "is" the Kronecker flow on the torus. In summary then-noting the fact that $\mathscr{F}_{\alpha} \cong \mathscr{F}_{1 / \alpha}$, which was established earlier - we have

$$
\mathscr{F}_{\alpha} \cong A_{\alpha} \otimes \mathscr{K} \cong C\left(\mathbb{T}^{2}\right) \times_{\beta, u} \mathbb{R}^{2} \cong C^{*}\left(\mathbb{R}^{2} \times \mathbb{Z}^{2}, \sigma\right)
$$

\section{§ 3.5. Introducing the Trace}

We have already noted that the algebra of Xia [34] is generated by operators with kernels $a \in C_{c}\left(\mathbb{T}^{2} \times \mathbb{R}^{2}\right), \omega=(\theta, \phi) \in \mathbb{T}^{2}$ and $f \in L^{2}\left(\mathbb{R}^{2}\right)$ where these act by

$$
\left(\pi_{\omega}(a) f\right)(x, y)=\int_{\mathbb{R}^{2}} a((\theta+x, \phi+y), \xi, \eta) e^{\imath \beta x \eta} f(x+\xi, y+\eta) d \xi d \eta
$$

The $C^{*}$-algebra generated by all the $\pi_{\omega}(a)$ for $a \in C c\left(\mathbb{T}^{2} \times \mathbb{R}^{2}\right)$ was denoted by $C_{\omega}^{*}\left(\mathbb{T}^{2}, \mathbb{R}^{2}, \beta\right)$. It contains the $C^{*}$-algebra $\mathscr{K}$ generated by operators of the form

$$
\int_{\mathbb{R}^{2}} b(\xi, \eta) \exp \left(i \xi D_{x}\right) \exp \left(i \eta D_{y}\right) d \xi d \eta
$$

where $b \in C_{c}\left(\mathbb{R}^{2}\right)$. The mapping of $D_{x}, D_{y}$ onto $\tilde{D}_{x}, \tilde{D}_{y}$ defined in the previous section extends to give an isomorphism of $\mathscr{K}$ with the compact operators on $L^{2}(\mathbb{R}){ }^{4}$

The algebra $C_{\omega}^{*}\left(\mathbb{T}^{2}, \mathbb{R}^{2}, \beta\right)$ is endowed with a trace via the isomorphism with $C *\left(\mathbb{T}^{2}, \mathbb{R}^{2}, \beta\right)$, where the trace on the latter is given by

$$
\tau\left(C_{a}\right)=\int_{\mathbb{T}^{2}} a(\omega, 0,0), \text { for } a \in C_{c}\left(\mathbb{T}^{2} \times \mathbb{R}^{2}\right) .
$$

The proof of Lemma 1.3 in [34] makes it clear that an element of $\mathscr{K}$ is $\tau$-trace class if and only if its image in $\mathscr{K}\left(L^{2}(\mathbb{R})\right)$ is of ordinary trace class: indeed, the two

${ }^{4}$ Note that this shows that the algebra of observables in the potential free situation is actually isomorphic to $\mathscr{K}\left(L^{2}(\mathbb{R})\right)$. 
traces are proportional. Thus if we consider exp $\left(-t H_{0}\right)$ for $H_{0}$ the Hamiltonian defined above, it is of $\tau$-trace class if and only if $\exp \left(-t \tilde{H}_{0}\right)$ is of ordinary trace class in $\mathscr{K}\left(L^{2}(\mathbb{R})\right)$, where $\tilde{H}_{0}$ denotes the transformed Hamiltonian. But $\tilde{H}_{0}$ is simply a one dimensional harmonic oscillator on $L^{2}(\mathbb{R})$, and so its spectrum is simply $\left\{|\beta|\left(n+\frac{1}{2}\right): n \in \mathbb{N}\right\}$, for $\beta$ a real constant. So for $|\beta|>0$ we have

$$
\begin{aligned}
\tau\left(\exp \left(-t H_{0}\right)\right) & \propto e^{-t|\beta| / 2} \frac{1}{1-e^{-|\beta| t}} \\
& =\frac{e^{|\beta| t / 2}}{e^{|\beta| t}-1} \text { for } t>0 .
\end{aligned}
$$

Thus $\exp \left(-t H_{0}\right)$ is of $\tau$-trace class, a result which implies that the resolvent of the Hamiltonian is also of trace class.

We can extend this result to the case in which a bounded potential $V$ is added to $H_{0}$ by invoking a generalized Golden-Thompson inequality due to Ruskai [30]. The trace $\tau$ defined above extends to the weak closure of the algebra to give a normal semifinite trace on the von Neumann algebra so defined. Given this, and noting the fact that $-t H_{0}$ is bounded above for any $t>0$, the result of interest is [30, Theorem 4]:

Theorem 2. If $A$ and $B$ are self-adjoint operators, bounded above, and $A+B$ is essentially self-adjoint then

$$
\tau\left(e^{A+B}\right) \leq \tau\left(e^{A / 2} e^{B} e^{A / 2}\right) .
$$

Further, if $\tau\left(e^{A}\right)<\infty$, or $\tau\left(e^{B}\right)<\infty$ then

$$
\tau\left(e^{A+B}\right) \leq \tau\left(e^{A} e^{B}\right) .
$$

Taking $A=-t H_{0}$ and $B=-t V$, we note that both $A$ and $B$ are bounded above. Furthermore $H_{0}+V$ is essentially self-adjoint [25], and so we have a pair of operators satisfying the requirements of the above theorem. The result obtained is

$$
\begin{aligned}
\left|\tau\left(\exp \left(-t\left(H_{0}+V\right)\right)\right)\right| & \leq\left|\tau\left(\exp \left(-t H_{0}\right) \exp (-t V)\right)\right| \\
& \leq|| \exp (-t V) \|\left|\tau\left(\exp \left(-t H_{0}\right)\right)\right| .
\end{aligned}
$$

That is $\exp \left(-t\left(H_{0}+V\right)\right)$ is of $\tau$ trace class $\forall t>0$, and the resolvent of the Hamiltonian with a bounded potential is also trace class. 


\section{§ 3.6. Projective Modules}

Consider now the Hamiltonian on $L^{2}\left(\mathbb{R}^{2}\right)$ that represents electrons moving in a plane subject to a perpendicular magnetic field:

$$
\begin{aligned}
H_{0} & =\frac{1}{2}\left(D_{x}^{2}+D_{y}^{2}\right) \\
& =\frac{1}{2}\left(\left(-i \partial_{x}+\frac{1}{2} \alpha y\right)^{2}+\left(-i \partial_{y}-\frac{1}{2} \alpha x\right)^{2}\right) \\
& =-\frac{1}{2}\left(\partial_{x}^{2}+\partial_{y}^{2}\right)+\frac{1}{8} \alpha^{2}\left(x^{2}+y^{2}\right)+\frac{i}{2} \alpha\left(x \partial_{y}-y \partial_{x}\right) .
\end{aligned}
$$

We show that if $\alpha$ is irrational then this Hamiltonian gives rise to representation of the irrational rotation algebra $A_{\alpha}$ which is contained in the commutant of the algebra of observables, and determine both the Chern number and the Murray-von Neumann dimension of the projective module so obtained.

It is well known that the Hamiltonian defined above is equivalent to that of a one dimensional harmonic oscillator. To see this explicitly, consider the following sequence of transformations. Let $V_{1}$ denote multiplication by $e^{i \alpha x y}$. Then the Fourier transforms of $V_{1} D_{x} V_{1}{ }^{*}$ and $V_{1} D_{y} V_{1}{ }^{*}$ have the following forms:

$$
\begin{aligned}
\left(V_{1}{\widehat{D_{x} V_{1}^{*}}}^{*}\right)(r, s) & =\int_{\mathbb{R}^{2}} e^{-2 \pi i(r x+s y)}\left(\left(-i \partial_{x}-\alpha y\right) f\right)(x, y) d x d y \\
& =2 \pi r \hat{f}(r, s)+\frac{\alpha}{2 \pi i} \partial_{s} \hat{f}(r, s) \\
\left(V_{1}{\widehat{D_{y} V_{1}}}^{*} f\right)(r, s) & =\int_{\mathbb{R}^{2}} e^{-2 \pi i(r s+s x)}\left(-i \partial_{y} f\right)(x, y) d x d y \\
& =2 \pi s \hat{f}(r, s):
\end{aligned}
$$

Finally as in [34], let $V_{2}$ denote multiplication by $\exp \left(i 2 \pi^{2} r s / \alpha\right)$, and define

$$
\tilde{D}_{x}=V_{2}\left(\widehat{V_{1}}{\widehat{D_{x}}}^{*}\right) V_{2}^{*} \text { and } \tilde{D}_{y}=V_{2}\left(\widehat{V_{1}}{\widehat{D_{y}}}^{*}\right) V_{2}^{*}
$$

Simple calculations show that these operators have the form of a one dimensional harmonic oscillator:

$$
\begin{aligned}
& \tilde{D}_{x}=\text { multiplication by } 2 \pi s \\
& \tilde{D}_{y}=\frac{\alpha}{2 \pi i} \times \text { differentiation by } s .
\end{aligned}
$$


That is, $\tilde{D}_{x}$ and $\tilde{D}_{y}$ act only on the second "component" in

$$
L^{2}\left(\mathbb{R}^{2}\right) \cong L^{2}(\mathbb{R}) \otimes L^{2}(\mathbb{R})
$$

Given this we consider the action of the "straightening operations" used above on the standard magnetic translation operators. Define then

$$
\left(\mathscr{U}_{m, n} f\right)(x, y)=e^{\pi i \alpha(m y-n x)} f(x+m, y+n),
$$

for $f \in L^{2}\left(\mathbb{R}^{2}\right)$ and $(m, n) \in \mathbb{Z}^{2}$. Easy calculations show that the for any $(m, n) \in \mathbb{Z}^{2}$ the operator $\mathscr{U}_{m, n}$ commutes with the Hamiltonian, ${ }^{5}$ and that

$$
\left(V_{1} \mathscr{U}_{m, n} V_{1}^{*} f\right)(x, y)=e^{-2 \pi i \alpha n x} e^{-\pi i \alpha m n} f(x+m, y+n)
$$

Writing $\tilde{\mathcal{U}}_{m, n}$ for $V_{2}\left(V_{1} \widehat{\mathcal{U}}_{m, n} V_{1}^{*}\right) V_{2}^{*}$ we obtain

$$
\left(\check{\mathscr{U}}_{m, n} f\right)(r, s)=e^{\pi i \alpha m n} e^{2 \pi i m} f(r+(\alpha n), s) .
$$

Specifically, the two generators are given by

$$
\begin{aligned}
& \left(\tilde{\mathscr{U}}_{1,0} f\right)(r, s)=e^{2 \pi t r} f(r, s) \\
& \left(\tilde{\mathscr{U}}_{0,1} f\right)(r, s)=f(r+\alpha, s) .
\end{aligned}
$$

So the action of the translations is also restricted to one of the variables when we write $L^{2}\left(\mathbb{R}^{2}\right) \cong L^{2}\left(\mathbb{R}^{2}\right) \otimes L^{2}(\mathbb{R})$ : the first variable, here, illustrating the fact that $\left[\mathscr{U}_{m, n}, H_{0}\right]=0$ in a particularly obvious manner.

What we've obtained then is a representation of the algebra $A_{\alpha}$ on $L^{2}(\mathbb{R})$, for notice that

$$
\tilde{\mathscr{U}}_{1,0} \tilde{\mathscr{U}}_{0,1}=e^{2 \pi i \alpha} \tilde{\mathscr{U}}_{0,1} \tilde{\mathscr{U}}_{1,0}
$$

Now Connes [9] gives explicit representatives for each element of $K_{0}\left(A_{\alpha}\right)$ for $\alpha$ irrational, considered as differences of finitely generated projective modules. They're indexed by two integers, $p, q$ say, and the total space of the module is $q$ copies of Schwarz space. If we label the module indexed by $p$ and $q$ by $\mathscr{E}_{p, q}$ then the

${ }^{5}$ Of course the commutativity does not depend upon $(m, n)$ being in $\mathbb{Z}^{2}$, for there's no potential term in the Hamiltonian to define a standard length for the system. However, redefining the basic magnetic translation operators simply corresponds to taking a different value of $\alpha$. Should the basic translations be of lengths $a$ and $b$ then $\alpha a b$ must be irrational for what follows. 
actions of $U$ and $V$, the two generators of $A_{\alpha}$, are

$$
\begin{aligned}
& (U \xi)(s, h)=\xi(s, h) e^{2 \pi i(s+h p / q)} \\
& (V \xi)(s, h)=\xi(s-\epsilon, h+1) .
\end{aligned}
$$

Here $\xi \in \mathscr{E}_{p, q}, h \in \mathbb{Z}_{q}, s \in \mathbb{R}$, and $\epsilon=p / q-\alpha$. Choosing $q=1$ and $p=0$ we obtain $\epsilon=$ $-\alpha$ and

$$
\begin{aligned}
& (U \xi)(s)=\xi(s) e^{2 \pi \imath s} \\
& (V \xi)(s)=\xi(s+\alpha),
\end{aligned}
$$

which are exactly the $\tilde{\mathscr{U}}_{1,0}$ and $\tilde{\mathscr{U}}_{0,1}$ actions from above. Thus the module determined by the Hamiltonian and the magnetic translations is, up to smoothness requirements, precisely $\mathscr{E}_{0,1}$.

The Murray-von Neumann dimension of the module $\mathscr{E}_{0,1}$ is simply [9]

$$
\int_{0}^{1}|p-q \phi| d t=|\alpha|
$$

Connes's work also allows the calculation of the Chern number (again assuming that $\alpha$ is irrational, for the calculation requires lines of slope $p / \bar{q}$ to be transversals of the Kronecker foliation of slope $\alpha$ ):

$$
c_{1}(\mathscr{E})=\frac{|-\alpha|}{-\alpha}= \begin{cases}1 & \text { if } \alpha<0, \\ -1 & \text { if } \alpha>0 .\end{cases}
$$

\section{§ 4. The Conductivity of the Discrete Model}

\section{$\S 4.1$. The Spectrum of the Discrete Hamiltonian}

Returning now to our discrete Hamiltonian, we recall that

$$
(H \lambda)_{m, n}=\lambda_{m+1, n}+\lambda_{m-1, n}+e^{2 \pi m a m} \lambda_{m, n+1}+e^{-2 \pi \alpha a m} \lambda_{m, n-1} .
$$

Fourier transforming on the $y$-variable by defining

$$
\left(\lambda\left(k_{y}\right)\right)_{m}=\sum_{n=-\infty}^{\infty} \exp \left(-2 \pi i k_{y} n\right) \lambda_{m, n}
$$

for $k_{y} \in(0,1]$, gives a family of Hamiltonians $H\left(k_{y}\right)$ parameterised by $k_{y}$ : 


$$
\begin{aligned}
\left(H\left(k_{y}\right) \lambda\right)_{m} & =\lambda_{m+1}+\lambda_{m-1}+\exp \left(2 \pi i\left(\alpha m+k_{y}\right)\right) \lambda_{m}+\exp \left(-2 \pi i\left(\alpha m+k_{y}\right)\right) \lambda_{m} \\
& =\lambda_{m+1}+\lambda_{m-1}+2 \cos \left(2 \pi\left(\alpha \mathrm{m}+k_{y}\right)\right) \lambda_{m}
\end{aligned}
$$

This is the "almost Mathieu operator" which has been studied extensively (see for instance $[6,32]$, and references therein). It may be interpreted as a onedimensional Schrödinger operator with potential $2 \cos \left(2 \pi\left(\alpha m+k_{y}\right)\right)$, by noting that the operator $\Delta_{d}$ defined by

$$
\left(\Delta_{d} \lambda\right)_{m}=\lambda_{m+1}-\lambda_{m-1}
$$

is the discrete analogue of the Laplacian. This identification is made clearer [12] by observing that

$$
\left\langle\lambda,-\Delta_{d} \lambda\right\rangle=\sum_{\substack{i<j \\|i-j|=1}}\left|\lambda_{i}-\lambda_{j}\right|^{2} .
$$

We note that the almost Mathieu equation also occurs in the context of the continuous model, when considering the effect of a weak periodic potential on a single Landau level [33]. In this case, however, the flux $\alpha$ in the above equation is replaced by $1 / \alpha$.

For $\alpha$ irrational and any $k_{y} \in(0,1]$, an irreducible, faithful representation of $A_{\alpha}$ on $l^{2}(\mathbb{Z})$ is obtained by setting

$$
(U \lambda)_{m}=\lambda_{m+1} \text { and }(V \lambda)_{m}=\exp \left(2 \pi i\left(\alpha m+k_{y}\right)\right) \lambda_{m}
$$

The simplicity of $A_{\alpha}$ for $\alpha$ irrational implies that the spectrum of $H$ in any of these representations is the same as that of $H=U+U^{*}+V+V^{*} \in A_{\alpha}$

Calculating the spectrum for irrational values of $\alpha$ is difficult. A proof that it is Cantor has been given in [6] for a dense $G_{\delta}$ of pairs $(\alpha, \chi) \in \mathbb{R}^{2}$, which were unspecified modulo the irrationality of $\alpha$. More recently, [8] provides a proof that the spectrum of $H(\alpha)$ for $\alpha$ an irrational Liouville number is a Cantor set. Recall that $\alpha$ is a Liouville number if

$$
\forall C>0 \exists p / q \in \mathbb{Q} \text { with }|\alpha-p / q|<C^{-q} .
$$

So we expect to see gaps in the spectrum of the Hamiltonian, and because $\alpha$ represents the physical magnetic field we'd expect that the gap boundaries would change continuously with respect to $\alpha$. This property is most easily proven by noting that $\left\{A_{\alpha}: \alpha \in \mathbb{T}\right\}$ are a continuous field of $C^{*}$-algebras, (see [13]). Of course it's necessary to stipulate an algebra of sections, and this is naturally provided by the "universal rotation algebra", hereafter denoted $\mathscr{A}$. This is the universal $C^{*}$-algebra generated by three unitaries $U, V$ and $W$ satisfying 


$$
U W=W U, \quad V W=W V \text {, and } U V=W V U
$$

Note that $W$ is in the centre of $\mathscr{A}$, and so maps to $\lambda 1$ (for $\lambda$ a scalar of modulus 1 ) in any irreducible representation of $\mathscr{A}$. The defining relations of the algebra then collapse to

$$
\pi(U) \pi(V)=\lambda \pi(V) \pi(U)
$$

so if $\lambda=e^{2 \pi i \alpha}$ we have a representation of the rotation algebra $A_{\alpha}$. More simply, we can define a $*$-homomorphism $\pi_{\lambda}: \mathscr{A} \rightarrow A_{\alpha}$ by

$$
\pi_{\lambda}(U)=U \text { and } \pi_{\lambda}(V)=V
$$

(so that $\left.\pi_{\lambda}(W)=\lambda 1\right)$ for any $\lambda \in \mathbb{T}$. That $\mathscr{A}$ is the algebra of sections that allows the identification of $\left\{A_{\alpha}: \alpha \in \mathbb{T}\right\}$ as a continuous field of $C^{*}$-algebras (implicit in [13]) follows from [1], which includes the following result.

Theorem 3. If $\lambda \mapsto x(\lambda)$ is a map of $\mathbb{T}$ such that each $x(\lambda)$ is in $A_{\alpha}$, for $\lambda=e^{2 \pi i \alpha}$, and for each $\epsilon>0$ and $\lambda_{0} \in \mathbb{T} \exists y \in \mathscr{A}$ such that

$$
\left\|x(\lambda)-\pi_{\lambda}(y)\right\|<\epsilon \forall \lambda \text { near } \lambda_{0}
$$

then $\exists x \in \mathscr{A}$ with $\pi_{\lambda}(x)=x(\lambda) \forall \lambda \in \mathbb{T}$.

The crucial consequences of viewing the rotation algebras in this way are that the maps $\lambda \mapsto\left\|\pi_{\lambda}(x)\right\|$ and $\lambda \mapsto \tau(\pi(x))$ are continuous for $x \in \mathscr{A}$. Furthermore the action of units is continuous, so by [13], if $X$ is open in $\mathbb{C}$ then $\{\lambda \in \mathbb{T}: \operatorname{sp}(a(\lambda))$ $\subseteq X\}$ is open, for $\lambda \mapsto a(\lambda)$ a continuous section such that $a(\lambda)$ is normal $\forall \lambda \in \mathbb{T}$. In particular, if we take

$$
a(\lambda)=H_{\lambda}=U+U^{*}+V+V^{*} \in A_{\lambda}
$$

then $a(\lambda)$ is self-adjoint.

Suppose now that there are sequences $\left\{\alpha_{n}\right\} \rightarrow \alpha$ and $\left\{E_{n}\right\} \rightarrow E$ such that $E_{n} \in \operatorname{sp}\left(H_{\alpha_{n}}\right) \forall n \in \mathbb{N}$. Then if $E \notin \operatorname{sp}\left(H_{\alpha}\right)$ there is an open set $X \subseteq \mathbb{C}$ containing $E$ such that

$$
\operatorname{sp}\left(H_{\alpha}\right) \cap X=\emptyset
$$

Thus we have that for some $\delta>0$, 


$$
\operatorname{sp}\left(H_{\hat{\alpha}}\right) \cap X=\emptyset \quad \forall \hat{\alpha} \text { such that }|\hat{\alpha}-\alpha|<\delta,
$$

contradicting the assumptions. So the gap boundaries of the spectrum vary continuously with changes in magnetic field.

The universal rotation algebra also provides a natural setting in which to examine the almost Mathieu operators considered earlier. Firstly note that $\mathscr{A} \cong$ $C^{*}($ Heis $(\mathbb{Z}))$, the group $C^{*}$-algebra corresponding to the integer Heisenberg group. Recall that the latter can be realised as a semi-direct product $\mathbb{Z}^{2} \times \mathbb{Z}$, where

$$
(m, n, p)(\bar{m}, \bar{n}, \bar{p})=(m+\bar{m}, n+\bar{n}, p+\bar{p}+m \bar{n}) .
$$

So the regular representation of $\operatorname{Heis}(\mathbb{Z})$ on $l^{2}\left(\mathbb{Z}^{3}\right)$ is given by

$$
(\pi(m, n, p) f)(\bar{m}, \bar{n}, \bar{p})=f(m+\bar{m}, n+\bar{n}, p+\bar{p}+m \bar{n}),
$$

and the three generators $U, V, W$ found in the abstract definition of the universal rotation algebra take the form

$$
\begin{aligned}
& (\pi(1,0,0) f)(\bar{m}, \bar{n}, \bar{p})=f(\bar{m}+1, \bar{n}, \bar{p}+\bar{n}) \\
& (\pi(0,1,0) f)(\bar{m}, \bar{n}, \bar{p})=f(\bar{m}, \bar{n},+1, \bar{p}) \\
& (\pi(0,0,1) f)(\bar{m}, \bar{n}, \bar{p})=f(\bar{m}, \bar{n}, \bar{p}+1) .
\end{aligned}
$$

Fourier transforming over $\bar{m}$ and $\bar{p}$ gives a family of representations over $\mathbb{T}^{2}$.

$$
\begin{aligned}
& (\pi(1,0,0) f)_{\theta, \phi}(\bar{n})=\exp (2 \pi i \phi \bar{n}) \exp (2 \pi i \theta) f_{\theta, \phi}(\bar{n}) \\
& (\pi(0,1,0) f)_{\theta, \phi}(\bar{n})=f_{\theta, \phi}(\bar{n}+1) \\
& (\pi(0,0,1) f)_{\theta, \phi}(\bar{n})=\exp (2 \pi i \phi) f_{\theta, \phi}(\bar{n}) .
\end{aligned}
$$

We define the "universal Hamiltonian" in $C^{*}(\operatorname{Heis}(\mathbb{Z}))$ to be

$$
\tilde{H}=U+V+U^{*}+V^{*},
$$

where $U \equiv \pi(1,0,0)$ and $V \equiv \pi(0,1,0)$. Then under the above mappings the $l^{2}\left(\mathbb{Z}^{3}\right)$ form of the Hamiltonian,

$$
(\tilde{H} \lambda)(m, n, p)=\lambda_{m+1, n, p+n}+\lambda_{m, n+1, p}+\lambda_{m-1, n, p-n}+\lambda_{m, n-1, p}
$$

becomes a two parameter family of Hamiltonians on $l^{2}(\mathbb{Z})$, indexed by $\theta$ and $\phi$ : 


$$
\begin{aligned}
\left(\tilde{H}_{\theta, \phi} f\right)(n) & =\exp (2 \pi i \theta) \exp (2 \pi i \phi n) f(n)+f(n+1)+\text { hermitian conjugate } \\
& =f(n+1)+f(n-1)+2 \cos (2 \pi(\theta+\phi n)) f(n) .
\end{aligned}
$$

Thus we have a direct integral decomposition,

$$
\tilde{H}=\int_{\mathbb{T}^{2}}^{\oplus} \tilde{H}_{\theta, \phi} d \mu(\theta, \phi) \text { on } \int_{\mathbb{T}^{2}}^{\oplus} l_{\theta, \phi}^{2}(\mathbb{Z}) d \mu(\theta, \phi)
$$

and recognise that $\tilde{H}_{\theta, \phi}$ is again the familiar "almost Mathieu" operator [6] considered above that arises directly from the discrete Hamiltonian. ${ }^{6}$

\section{§ 4.2. Conductivity for the Discrete Model}

We outline a proof that the Hall conductance for the discrete model is given by the Chern number of the projection onto the Fermi level when the latter lies in a spectral gap. Rather than mimic the proof provided in the continuous case by Xia [34, Lemma 3.1], which is certainly possible, we use the well known properties of projections in the rotation algebra, together with a formula established in 1982 by Streda [31], which relates the Hall conductivity to the derivative of the density of states with respect to the magnetic field. ${ }^{7}$

More precisely, Středa worked from the ubiquitous Kubo formula to obtain:

$$
\sigma_{H}=\frac{e^{2}}{h} \frac{\partial}{\partial \alpha} \tau\left(P_{F}\right)(\alpha),
$$

where the derivative is taken with $E_{F}$, the Fermi energy, fixed. Of course we need to check that this is well defined for the case in hand, so suppose that for a given

${ }^{6}$ Note that in [6] Bellissard and Simon are really considering $H_{\phi}=\int_{\mathbb{T}}^{\oplus} \tilde{H}_{\theta . \phi} d \mu(\theta)$, which explains the definition of the spectrum as the union over all the values of $\theta$. For $\phi$ irrational the spectrum of $\tilde{H}_{\theta, \phi}$ is independent of $\theta$, but for $\phi$ rational the spectrum of $\tilde{H}_{\theta, \phi}$ is of course just a set of $q$ points (counting multiplicities) with a continuous dependence on $\theta$. As mentioned earlier, the representation outlined above which takes $\tilde{H}$ to $\int_{\mathbb{T}}^{\oplus} \tilde{H}_{\theta, \phi} d \mu(\theta)$ is faithful, so the union of the spectra of the $\tilde{H}_{\theta, \phi}$ over $\theta$ gives the spectrum of the element $U+V+U^{*}+V^{*}$ in the $C^{*}$-algebra $A_{\phi}$.

'Although Streda's formula is derived for a continuous Hamiltonian, it is easily seen to hold for the case in hand. Indeed, as pointed out by Hadju et al. in [19], Středa implicitly assumes that the trace of the projection is uniformly bounded when he interchanges two limits, and whilst this is not a problem for either a confined system (as considered in [19]) or for a discrete system as we are considering here, it requires further justification in the case of particles moving in $\mathbb{R}^{2}$ without a confining potential. 
$\alpha^{\prime}$ the projection $P_{F}$ lies in a gap of the spectrum of the discrete Hamiltonian. Recall that $K_{0}\left(A_{\alpha}\right) \cong \mathbb{Z} \oplus \mathbb{Z}$, and is generated by the class of the identity projection, [1], and $\left[e_{R}\right]$, the class of the Powers-Rieffel projection [27]. So we can write

$$
\left[P_{F}\right]=m[1]+n\left[e_{R}\right]
$$

for some $m, n \in \mathbb{Z}$. Thus we can determine the trace of the projection:

$$
\tau\left(P_{F}\right)=m+n \alpha^{\prime}
$$

From the above discussion of the $A_{\alpha}$ 's as a continuous field of $C^{*}$-algebras, the gap persists for all $\alpha$ in the open interval $\left(\alpha^{\prime}-\delta, \alpha^{\prime}+\delta\right)$ for some $\delta>0$. The section

$$
\alpha \mapsto P_{F}(\alpha) \in A_{\alpha}
$$

is a continuous section of the continuous field $\left(A_{\alpha}\right)_{\alpha \in\left[\alpha^{\prime}-\delta / 2, \alpha^{\prime}+\delta / 2\right]}$ by [14], and consequently the Chern number $n$ of $P_{F}(\alpha)$ is constant for $\alpha \in\left[\alpha^{\prime}-\delta / 2, \alpha^{\prime}+\delta / 2\right]$. That is, for $\alpha$ in this range,

$$
\tau\left(P_{F}\right)(\alpha)=\tau\left(m[1]+n\left[e_{R}(\alpha)\right]\right)=m+n \alpha .
$$

Note that $m$ is constant because $\tau\left(P_{F}\right)(\alpha)$ is required to lie between 0 and 1 . Thus we have, suppressing the $\alpha$ dependence of the $P_{F}(\alpha)$,

$$
\sigma_{H}=\frac{e^{2}}{h} \frac{\partial}{\partial \alpha} \tau\left(P_{F}\right)=\frac{e^{2}}{h} n=\frac{e^{2}}{h} \frac{1}{2 \pi i} \tau\left(P_{F}\left[\delta_{1}\left(P_{F}\right), \delta_{2}\left(P_{F}\right)\right]\right)
$$

and the stability of the conductance with respect to changes in the magnetic field is clear from the proof.

\section{§4.3. Comparison with the Formula for Rational Flux}

We demonstrate the reduction of this formula to that used by Kohmoto and Fradkin for rational values of the flux. Define a Fourier tansform on $q \mathbb{Z}$ as follows. For $m \in \mathbb{Z}$ write $m=s q+r$ where $0 \leq r<q-1$ and put

$$
\left(\lambda\left(k_{x}\right)\right)_{r}=\sum_{s=-\infty}^{\infty} \exp \left(i q k_{x} s\right) \lambda_{s q+r} \text { for } k_{x} \in(0,1 / q]
$$

Simple calculations show that 


$$
\begin{aligned}
(\widehat{U \lambda})_{r}\left(k_{x}, k_{y}\right) & =\sum_{s \in \mathbb{Z}} \exp \left(-2 \pi i q k_{x} s\right) \lambda_{s q+r+1}\left(k_{y}\right) \\
& = \begin{cases}\hat{\lambda}_{r+1}\left(k_{x}, k_{y}\right) & \text { if } r+1<q \\
\exp \left(2 \pi i q k_{x}\right) \hat{\lambda}_{0}\left(k_{x}, k_{y}\right) & \text { if } r=q-1\end{cases} \\
(\widehat{V \lambda})_{r}\left(k_{x}, k_{y}\right) & =\exp \left(2 \pi i k_{y}\right) e^{2 \pi i p r / q} \hat{\lambda}_{r}\left(k_{x}, k_{y}\right),
\end{aligned}
$$

and so transforming the Hamiltonians used above gives a decomposition of $H$ into a two parameter family $H\left(k_{x}, k_{y}\right)$ of $q \times q$ matrices. We assume that $H\left(k_{x}, k_{y}\right)$ is non-degenerate for every $\left(k_{x}, k_{y}\right)$ in the MBZ. ${ }^{8}$ Given this, and dropping the explicit $\left(k_{x}, k_{y}\right)$ dependence of the operators herein, we have that for $n=1, \ldots, q$,

$$
e_{n} H=H e_{n}=\lambda_{n} e_{n}
$$

where $e_{n}$ is the eigenprojection corresponding to the $n$-th eigenvalue $\lambda_{n}$ of $H$. Applying the derivations $\delta_{i}$ to this equation yields

$$
\delta_{i}\left(e_{n}\right) H+e_{n} \delta_{i}(H)=\lambda_{n} \delta_{i}\left(e_{n}\right),
$$

and rearranging this expression after postmultiplication by $e_{m}$ for $m \neq n$ gives

$$
\delta_{i}\left(e_{n}\right) e_{m}=\frac{\delta_{i}(H) e_{m}}{\lambda_{n}-\lambda_{m}}
$$

Now note that the equation $\delta_{i}\left(e_{m}^{2}\right)=\delta_{i}\left(e_{m}\right)$ implies that

$$
e_{m} \delta_{i}\left(e_{m}\right) e_{m}=0
$$

so we have

$$
\begin{aligned}
e_{n} \delta_{i}\left(e_{n}\right) & =e_{n} \delta_{i}\left(e_{n}\right)\left\{\sum_{m \neq n} e_{m}\right\} \\
& =e_{n} \sum_{m \neq n} \frac{\delta_{i}(H) e_{m}}{\lambda_{n}-\lambda_{m}} .
\end{aligned}
$$

Suppose now that the Fermi projection $P_{F}$ lies in the gap between the $n$th and $(n$ +1 )st bands of the spectrum of the Hamiltonian. Then the Chern number of $P_{F}$ is given by

\footnotetext{
${ }^{8}$ That this assumption is reasonable follows because degeneracies occur only when the two central sub-bands meet, which happens [8] when $\alpha$ has an even denominator. But of course the assumption here is weaker in that we only need the $q$ eigenvalues of the $q \times q$ matrix above any point of the torus to be non-degenerate.
} 


$$
\begin{aligned}
c_{1}\left(P_{F}\right) & =\frac{1}{2 \pi i} \tau\left(P_{F}\left[\delta_{1}\left(P_{F}\right), \delta_{2}\left(P_{F}\right)\right]\right) \\
& =\frac{1}{2 \pi i} \tau \sum_{j=1}^{n} \sum_{m \neq j}\left(\frac{e_{j} \delta_{1}(H) e_{m} \delta_{2}\left(e_{j}\right)}{\left(\lambda_{j}-\lambda_{m}\right)}-\frac{\delta_{2}(H) e_{m} \delta_{1}\left(e_{j}\right)}{\left(\lambda_{j}-\lambda_{m}\right)}\right) \\
& =\frac{1}{2 \pi i} \tau \sum_{j=1}^{n} \sum_{m \neq j}\left(\frac{e_{j} \delta_{1}(H) e_{m} \delta_{2}(H) e_{j}}{\left(\lambda_{j}-\lambda_{m}\right)^{2}}-\frac{e_{j} \delta_{2}(H) e_{m} \delta_{1}(H) e_{j}}{\left(\lambda_{j}-\lambda_{m}\right)^{2}}\right) .
\end{aligned}
$$

Recall that $\delta_{1}(H)=2 \pi i\left(U-U^{*}\right)$ and $\delta_{2}(H)=2 \pi i\left(V-V^{*}\right)$, so if we have normalized eigenvectors $\phi_{m}$ for $m=1, \ldots, q$ the above expression for $c_{1}\left(P_{F}\right)$ equals

$$
-2 \pi \int_{0}^{1 / q} \int_{0}^{1} \sum_{j=1}^{n} \sum_{m \neq j} \frac{\left\langle\phi_{j} \mid\left(U-U^{*}\right) \phi_{m}\right\rangle\left\langle\phi_{m} \mid\left(V-V^{*}\right) \phi_{j}\right\rangle-\{U \leftrightarrow V\}}{\left(\lambda_{j}-\lambda_{m}\right)^{2}}
$$

where $\{U \leftrightarrow V\}$ indicates an identical term with $U$ and $U^{*}$ interchanged with $V$ and $V^{*}$ respectively. Here we've implemented the normalized trace $\tau$ in this representation of the rational rotation algebra, which is simply integration over the MBZ coupled with the usual matrix trace. Note that the terms in the sum with $m$ $<n$ clearly cancel, so the expression simplifies to

$$
c_{1}\left(P_{F}\right)=2 \pi \hbar^{2} \int_{0}^{1 / q} \int_{0}^{1} \sum_{m \leq n<j}\left(\frac{\left(v_{x}\right)_{j m}\left(v_{y}\right)_{m j}-\left(v_{y}\right)_{j m}\left(v_{x}\right)_{m I}}{\left(\lambda_{j}-\lambda_{m}\right)^{2}}\right),
$$

which, modulo the $e^{2} / h$ that represents the basic unit of conductance, is precisely the expression for the conductance obtained by Kohmoto and Fradkin [15] that was cited in $\S 2$.

\section{§ 5. Fredholm Modules and Analytical Indices}

We define a Fredholm module for the algebra of observables of the discrete model that plays a similar role to that defined by Bellissard for the continuum model of the quantum Hall effect. We first recall the following definiton from [11].

Definition 2. A p-summable Fredholm module over an associative algebra $A$ is a pair $(\mathscr{H}, F)$, where

1. $\mathscr{H}=\mathscr{H}^{+} \oplus \mathscr{H}^{-}$is a $\mathbb{Z}_{2}$ graded Hilbert space with grading operator $\epsilon$.

2. $\mathscr{H}$ is a left $A$-module: ${ }^{9}$ that is, $\exists$ homomorphisms $\pi^{+}, \pi^{-}: A \rightarrow B\left(\mathscr{H}^{ \pm}\right)$such that

\footnotetext{
${ }^{9}$ For the sake of simplicity we assume $A$ to be trivially $\mathbb{Z}^{2}$-graded here.
} 
the map

$$
\pi: a \mapsto\left[\begin{array}{cc}
\pi^{+}(a) & 0 \\
0 & \pi^{-}(a)
\end{array}\right]
$$

is a representation of $A$ on $\mathscr{H}$.

3. $F \in B(\mathscr{H}), F^{2}-1=0, F \epsilon=-\epsilon F$ and for any $a \in A$

$$
F \pi(a)-\pi(a) F \in \mathscr{L}^{P}(\mathscr{H})
$$

Note that $\mathscr{L}^{p}(\mathscr{H})$ is the Schatten ideal in $B(\mathscr{H})$, defined by

$$
\mathscr{L}^{p}(\mathscr{H})=\left\{T \in B(\mathscr{H}): \text { Trace }|T|^{p}<\infty\right\}
$$

\section{§ 5.1. A Fredholm Module for the Discrete Model}

The obvious means of generating a Fredholm module for the discrete model - mimicking the continuum construction by replacing $\mathscr{H}=L^{2}\left(\mathbb{R}^{2}\right) \oplus L^{2}\left(\mathbb{R}^{2}\right)$ by $\mathscr{H}=$ $l^{2}\left(\mathbb{Z}^{2}\right) \oplus l^{2}\left(\mathbb{Z}^{2}\right)$ and taking $F$ to be the discrete analogue of Bellissard's $F$ operatoris certainly possible, ${ }^{10}$ and has recently been published [5]. We choose another, more transparent, approach, and extend Connes's construction of a $p>2$-summable module for $C\left(\mathbb{T}^{2}\right)$ to all non-commutative tori, by viewing the latter as $C^{*}\left(\mathbb{Z}^{2}, \gamma\right)$ and eliminating all reference to an underlying (commutative) space.

Consider then $A_{\alpha}$ as $C^{*}\left(\mathbb{Z}^{2}, \gamma\right)$, for $\gamma((m, n),(\bar{m}, \bar{n}))=\exp (\pi i \alpha(m \bar{n}-\bar{m} n)$. Standard calculations yield a representation of the algebra on $l^{2}\left(\mathbb{Z}^{2}\right)$ via

$$
\begin{aligned}
& (U \lambda)(m, n)=e^{\pi \alpha n} \lambda(m-1, n) \\
& (U \lambda)(m, n)=e^{-\pi \alpha m} \lambda(m, n-1) .
\end{aligned}
$$

The smooth subalgebra in which we are interested is just the set of elements $a=\sum_{(m, n) \in \mathbb{Z}^{2}} a_{m, n} U^{m} V^{n}$ of $A_{\alpha}$ for which $\delta_{1}^{\gamma} \delta_{2}^{s} a$ is in $A_{\alpha}$ for all $(r, s) \in \mathbb{Z}^{2}$. That is, $[9],\left\{a_{m, n}\right\}$ must be a rapidly decreasing sequence. Now take $\mathscr{H}=\mathscr{H}^{+} \oplus \mathscr{H}^{-}$for

$$
\mathscr{H}^{-}=l^{2}\left(\mathbb{Z}^{2}\right), \mathscr{H}^{+}=\left\{\lambda \in l^{2}\left(\mathbb{Z}^{2}\right): \bar{\partial} \lambda \in l^{2}\left(\mathbb{Z}^{2}\right)\right\},
$$

\footnotetext{
${ }^{10} \mathrm{We}$ add the caveat that Bellissard et al. seem to have ignored the singularity in the definition of the $F$ operator at $(0,0)$, and whilst this is irrelevant in the continuum case, it is no longer so for the discrete construction.
} 
where $\bar{\partial} \lambda(m, n)=i(m+i n) \lambda(m, n)$, and define

$$
F=\left[\begin{array}{cc}
0 & (\bar{\partial}+\epsilon)^{-1} \\
(\bar{\partial}+\epsilon) & 0
\end{array}\right] .
$$

Here $\epsilon$ is any complex number such that $i(m+i n)+\epsilon \neq 0 \forall(m, n) \in \mathbb{Z}^{2}$. Then letting

$$
\Theta_{m, n}^{-}(g, h)=\delta_{m, g} \delta_{n, h}
$$

denote the standard basis of $l^{2}\left(\mathbb{Z}^{2}\right)=\mathscr{H}^{-}$, and

$$
\Theta_{m, n}^{+}(g, h)=\frac{1}{i(m+i n)+\epsilon} \delta_{m, g} \delta_{n, h}
$$

the corresponding basis of $\mathscr{H}^{+}$, a short calculation reveals that

$$
U \Theta_{m, n}^{-}=e^{\pi i \alpha n} \Theta_{m+1, n}^{-} \text {and } V \Theta_{m, n}^{-}=e^{-\pi i \alpha m} \Theta_{m, n+1}^{-}
$$

Note that the operators are additive on the indices. If we define $\mathscr{U}_{m, n}$ to be $U^{m} V^{n}$ then

$$
\mathscr{U}_{m, n} \mathscr{U}_{\bar{m}, \bar{n}}=\exp (-2 \pi i \alpha n \bar{m}) \mathscr{U}_{m+\bar{m}, n+\bar{n}}
$$

and it's easily shown that

$$
\begin{aligned}
& \mathcal{U}_{\bar{m}, \bar{n}} \Theta_{m, n}^{-}=\exp (\pi i \alpha(n \bar{m}+\bar{n} \bar{m}-m \bar{n})) \Theta_{m+\bar{m}, n+\bar{n}}^{-} \\
& \mathcal{U}_{m, n} \Theta_{m, n}^{+}=\frac{i((m+\bar{m})+i(n+\bar{n}))+\epsilon}{i(m+i n)+\epsilon} \exp (\pi i \alpha(n \bar{m}-m \bar{n}+\bar{m} \bar{n})) \Theta_{m+\bar{m}, n+\bar{n}}^{+} .
\end{aligned}
$$

In order to compactify the equations to follow we denote the combinations of the form $m+i n$ by $\mathbf{m}$, with similar abbreviations for subscripted versions of the same combination.

Note that whilst the $\Theta_{m, n}^{+}$are not orthonormal as they stand, a straightforward calculation performed below (see the appendix to this section) reveals that the following result does not depend on the normality of the vectors, but merely their orthogonality. This is inherited directly from the orthogonality of the $\Theta_{m, n}^{-}$. So we suppress the normalization factors in the interests of readability.

Identifying $\Theta_{m, n}^{+}$with $\left(\Theta_{m, n}^{+}, 0\right) \in \mathscr{H}$, and noticing that

$$
F \Theta_{m, n}^{+}=(\partial+\epsilon) \Theta_{m, n}^{+}=\Theta_{m, n}^{-}
$$


we obtain

$$
\begin{aligned}
{\left[F, \mathscr{U}_{m, n}\right] \Theta_{m, n}^{+} } & =F \exp (\pi i \phi(\bar{m} n-m \bar{n}+\bar{m} \bar{n})) \frac{i(\mathbf{m}+\overline{\mathbf{m}})+\epsilon}{i \mathbf{m}+\epsilon} \Theta_{m+\bar{m}, n+\bar{n}}^{+}-\mathscr{U}_{\bar{m}, \bar{n}} \Theta_{m, n}^{-} \\
& =\exp (\pi i \phi(\bar{m} n-m \bar{n}+\bar{m} \bar{n}))\left\{\frac{i((\mathbf{m}+\overline{\mathbf{m}})+\epsilon}{i \mathbf{m}+\epsilon}-1\right\} \Theta_{m+\bar{m}, n+\bar{n}}^{-} \\
& =\exp (\pi i \phi(\bar{m} n-m \bar{n}+\bar{m} \bar{n})) \frac{i \overline{\mathbf{m}}}{i \mathbf{m}+\epsilon} \Theta_{m+\bar{m}, n+\bar{n}}^{-},
\end{aligned}
$$

and a similar calculation gives

$$
\left[F, \mathscr{U}_{\bar{m}, \bar{n}}\right] \Theta_{m, n}^{-}=-\exp (\pi i \phi(\bar{m} n-m \bar{n}+\bar{m} \bar{n})) \frac{i \overline{\mathbf{m}}}{i \mathbf{m}+\epsilon} \Theta_{m+\bar{m}, n+\bar{n}}^{+} .
$$

The main result of this paper is the following.

Theorem 4. The pair ( $\mathscr{H}, F)$ outlined above constitutes a p-summable Fredholm module over $A_{\alpha}^{\infty}$ for any $p>2$.

Proof. If we set $T_{m, n}=\left[F, \mathscr{U}_{m, n}\right]$, then we need find all $p \geq 1$ such that $\left[F, \Sigma a_{m, n} T_{m, n}\right] \in \mathscr{L}^{p}(\mathscr{H})$ for all rapidly decreasing sequences $\left\{a_{m, n}\right\}$. Recall that $\mathscr{L}^{p}(\mathscr{H})$ is a Banach space under the norm

$$
\left\|T_{m, n}\right\|_{\mathcal{L}_{p}}=\left(\sum_{j}\left(\mu_{j}\left(T_{m, n}\right)\right)^{p}\right)^{\frac{1}{p}},
$$

where the $\mu_{j}\left(T_{m, n}\right)$ are the singular values of $T_{m, n}$. Calculation shows that

$$
\begin{aligned}
\left\|T_{m, n}\right\|_{L^{p}}^{p} & =\sum_{(r, s) \in \mathbb{Z}^{2}} \frac{|m+i n|^{p}}{|(r-m)+i(s-n)-i \epsilon|^{p}} \\
& =|m+i n|^{p} \sum_{(r, s) \in \mathbb{Z}^{2}} \frac{1}{|r+i s-i \epsilon|^{p}},
\end{aligned}
$$

and simple considerations show that the final sum is finite if and only if $p$ is such that

$$
c=\sum_{r, s>0} \frac{1}{\left(r^{2}+s^{2}\right)^{p / 2}}<\infty .
$$

Now note that

$$
c=\sum_{k=2}^{\infty} \sum_{r+s=k} \frac{1}{\left(r^{2}+s^{2}\right)^{p / 2}}
$$

and consider the following inequalities: 


$$
2\left(\frac{k}{2}\right)^{2} \leq r^{2}+s^{2} \leq 2 k^{2}
$$

which give

$$
\left(\frac{1}{2 k^{2}}\right)^{p / 2} \leq \frac{1}{\left(r^{2}+s^{2}\right)^{p / 2}} \leq\left(\frac{2}{k^{2}}\right)^{p / 2}
$$

But there are $k-1$ pairs satisfying $m+n=k$, and so

$$
\frac{k-1}{\left(2 k^{2}\right)^{p / 2}} \leq \sum_{r+s=k} \frac{1}{\left(r^{2}+s^{2}\right)^{p / 2}} \leq(k-1)\left(\frac{2}{k^{2}}\right)^{p / 2}
$$

Now the left and right hand sides are of order $k^{-(p-1)}$ for large $k$, so the sum over $k$ converges if $p-1>1$. That is, $T_{m, n} \in \mathscr{L}^{p}(\mathscr{H}) \forall(m, n) \in \mathbb{Z}^{2}$ if $p>2$.

Now consider the series $\sum a_{m, n} T_{m, n}$, and write

$$
V_{r}=\sum_{|m+i n|<r} a_{m, n} T_{m, n} \text { for } r \in \mathbb{N}
$$

We have

$$
\left\|V_{s}-V_{r}\right\|_{\mathscr{L}_{p}} \leq \sum_{r<\left|m^{\perp} i n\right|<s}\left|a_{m, n}\right|\left\|T_{m, n}\right\|_{\mathscr{L}_{p}}
$$

for any $r, s \in \mathbb{N}$ with $s>r$. Using the formula for $\left\|T_{m, n}\right\|_{\mathscr{L}_{p}}$ found above we have

$$
\left\|V_{s}-V_{r}\right\|_{\mathscr{L}_{p}} \leq \sum_{r<|m+i n|<s} b\left|a_{m, n}\right||m+i n|,
$$

where $b$ is a constant independent of $(m, n)$. Thus $\left\|V_{s}-V_{r}\right\|_{\mathscr{L}_{p} \rightarrow 0 \text { as } r, s}$ $\rightarrow \infty$ (note that $a_{m, n}$ is of rapid decrease), and since $\mathscr{L}^{p}(\mathscr{H})$ is a Banach space we have $\sum a_{m, n} T_{m, n} \in \mathscr{L}^{p}(\mathscr{H})$ for $p>2$. That is, $(\mathscr{H}, F)$ is a $p$-summable Fredholm module for the algebra $A_{\alpha}^{\infty}$ for any $p>2$.

Theorem 5. If $\mathscr{U}_{\bar{m}, \tilde{n}}$ denotes $U^{\tilde{m}} V^{\bar{n}} \in A_{\alpha}^{\infty}$ then the character $\tau$ associated to the Fredholm module $(\mathscr{H}, F)$ found above is given by

$$
\tau\left(\mathscr{U}_{\bar{m}_{0}, \bar{n}_{0}}, \mathscr{U}_{\bar{m}_{1}, \bar{n}_{1}}, \mathscr{U}_{\bar{m}_{2}, \bar{n}_{2}}\right)=\exp \left(2 \pi i \alpha\left(\bar{n}_{1} \bar{m}_{1}+\bar{n}_{2} \bar{m}_{1}+\bar{n}_{2} \bar{m}_{2}\right)\right)(2 \pi i)^{2}\left(\bar{n}_{2} \bar{m}_{1}-\bar{m}_{2} \bar{n}_{1}\right) .
$$

Proof. In order to calculate the character of $(\mathscr{H}, F)$ we first consider the combination $\mathscr{F} \Theta_{m, n}^{+}$, defined by

$$
\mathscr{F} \Theta_{m, n}^{+}=\left[F, \mathscr{U}_{\bar{m}_{0,}, \bar{n}_{0}}\right]\left[F, \mathscr{U}_{\tilde{m}_{1}, \bar{n}_{1}}\right]\left[F, \mathscr{U}_{m_{2}, \bar{n}_{2}}\right] \Theta_{m, n}^{+} .
$$


A simple calculation shows that

$$
\begin{aligned}
& \mathscr{F} \Theta_{m, n}^{+} \\
& =\exp (\pi i \phi \Delta) \frac{i \overline{\mathbf{m}}_{0}}{i\left(\mathbf{m}+\overline{\mathbf{m}}_{2}+\overline{\mathbf{m}}_{1}\right)+\epsilon} \times \frac{-i \overline{\mathbf{m}}_{1}}{i\left(\mathbf{m}+\overline{\mathbf{m}}_{2}\right)+\epsilon} \times \frac{i \overline{\mathbf{m}}_{2}}{i \mathbf{m}+\epsilon} \Theta_{m+\tilde{m}_{2}+\tilde{m}_{1}+\tilde{m}_{0, n}+\tilde{n}_{2}+\tilde{n}_{1}+\tilde{n}_{0},}^{-}
\end{aligned}
$$

where $\Delta=n\left(\bar{m}_{0}+\bar{m}_{1}+\bar{m}_{2}\right)-m\left(\bar{n}_{0}+\bar{n}_{1}+\bar{n}_{2}\right)+\bar{m}_{0}\left(\bar{n}_{2}+\bar{n}_{1}+\bar{n}_{0}\right)-\bar{n}_{0}\left(\bar{m}_{2}+\bar{m}_{1}\right)+\bar{m}_{1} \bar{n}_{2}$ $-\bar{m}_{2} \bar{n}_{1}+\bar{m}_{1} \bar{n}_{1}+\bar{m}_{2} \bar{n}_{2}$, and the reason for bracketing the terms in this manner will soon be apparent.

The character is given by

$$
-1 / 2 \operatorname{Trace}\left(\epsilon F\left[F, \mathscr{U}_{\bar{m}_{0} \bar{n}_{0}}\right]\left[F, \mathscr{U}_{\bar{m}_{1} \bar{n}_{1}}\right]\left[F, \mathscr{U}_{\bar{m}_{2} \bar{n}_{2}}\right]\right),
$$

where the trace is the usual one on $\mathscr{B}(\mathscr{H})$. We henceforth denote this quantity by $\tau(0,1,2)$. Notice that the initial $F$ changes the $\Theta_{m, n}^{-}$in the above expression for $\mathscr{F} \Theta_{m, n}^{+}$to an $\Theta_{m, n}^{+}$, and that, modulo the normalization convention mentioned earlier,

$$
\left\langle\Theta_{m, n}^{+}, \Theta_{m+\tilde{m}_{2}+\tilde{m}_{1}+\tilde{m}_{0}, n+\tilde{n}_{2}+\tilde{n}_{1}+\tilde{n}_{0}}^{+}\right\rangle=\delta_{m, m+\bar{m}_{2}+\bar{m}_{1}+\bar{m}_{0}} \delta_{n, n+\tilde{n}_{2}+\tilde{n}_{1}+\bar{n}_{0}} .
$$

Similar expressions hold for the $\Theta_{m, n}^{-}$, and the action of the grading operator ensures that the contributions add upon taking the trace. Thus we have,

$$
\begin{aligned}
\tau(0,1,2)= & 2 \pi i \sum_{(m, n) \in \mathbb{Z}^{2}} \frac{i \overline{\mathbf{m}}_{0}}{i\left(\mathbf{m}+\overline{\mathbf{m}}_{2}+\overline{\mathbf{m}}_{1}\right)+\epsilon} \times \frac{-i \overline{\mathbf{m}}_{1}}{i\left(\mathbf{m}+\overline{\mathbf{m}}_{2}\right)+\epsilon} \times \frac{i \overline{\mathbf{m}}_{2}}{i \mathbf{m}+\epsilon} \\
& \cdot \exp \left(2 \pi i \alpha\left(\bar{n}_{1} \bar{m}_{1}+\bar{n}_{2} \bar{m}_{1}+\bar{n}_{2} \bar{m}_{2}\right)\right) .
\end{aligned}
$$

The fact that the exponential factor is independent of $(m, n)$ allows us to follow Connes's calculation for the case of $C\left(T^{2}\right)$, and evaluate the sum using Eisenstein series as in $[11]$. This gives

$$
\tau(0,1,2)=\exp \left(2 \pi i \alpha\left(\bar{n}_{1} \bar{m}_{1}+\bar{n}_{2} \bar{m}_{1}+\bar{n}_{2} \bar{m}_{2}\right)\right)(2 \pi i)^{2}\left(\bar{n}_{2} \bar{m}_{1}-\bar{m}_{2} \bar{n}_{1}\right)
$$

\section{§ 5.2. The Discrete Analogue of the Connes-Kubo Formula}

In order to derive the desired formula, recall the $\mathbb{R}^{2}$ action on $A_{\alpha}$ defined in $\S 2.2$, and the corresponding pair of derivations $\delta_{1}$ and $\delta_{2}$. These define [11] a complex

$$
\Omega=A_{\alpha}^{\infty} \otimes \Lambda\left(\operatorname{Lie}\left(\mathbb{R}^{2}\right)\right)^{*}
$$

If we let $\epsilon_{1}, \epsilon_{2}$ denote the canonical basis for $\left(\operatorname{Lie}\left(\mathbb{R}^{2}\right)\right)^{*}$, then 


$$
d: A_{\alpha}^{\infty} \rightarrow A_{\alpha}^{\infty} \otimes \Lambda^{1}\left(\operatorname{Lie}\left(\mathbb{R}^{2}\right)\right)^{*}
$$

is given by

$$
d a=\left(\delta_{1} a\right) \otimes \epsilon_{1}+\left(\delta_{2} a\right) \otimes \epsilon_{2}
$$

Notice that the unique normalized trace $\tau$ on $A_{\alpha}$ [27] extends to define an "integral" on $A_{\alpha}^{\infty} \otimes \Lambda^{2}\left(\operatorname{Lie}\left(\mathbb{R}^{2}\right)\right)$ * in the obvious manner:

$$
\int a \otimes \epsilon_{1} \wedge \epsilon_{2}=\tau(a)
$$

Furthermore, if we extend $d$ to the complex $\Omega=\Omega^{0} \oplus \Omega^{1} \oplus \Omega^{2}$ via

$$
d\left(a \otimes \epsilon_{1}+b \otimes \epsilon_{2}\right)=\left(\delta_{1}(b)-\left(\delta_{2}(a)\right) \otimes \epsilon_{1} \wedge \epsilon_{2}\right.
$$

and $d\left(a \otimes \epsilon_{1} \wedge \epsilon_{2}\right)=0$, and note that if $\omega \in \Omega^{1}$ then $\int d \omega=0$ (because the derivations $\delta_{1}$ and $\delta_{2}$ annihilate the identity), it's clear that the triple $(\Omega, d, \delta)$ is a 2-cycle in the sense of $[11]$.

Proposition 4. The character of the cycle $(\Omega, d, S)$ is equal to that of the cycle associated with the $p>2$ Fredholm module $(\mathscr{H}, F)$ defined above.

Proof. The derivations act on $\mathscr{U}_{\bar{m}, \tilde{n}}=U^{\bar{m}} V^{\bar{n}}$ by

$$
\delta_{1} \mathscr{U}_{\bar{m}, \bar{n}}=2 \pi i \bar{m} \mathscr{U}_{\bar{m}, \bar{n}} \text { and } \delta_{2} \mathscr{U}_{\bar{m}, \bar{n}}=2 \pi i \bar{n} \mathscr{U}_{\bar{m}, \bar{n}},
$$

and thus we have

$$
\mathcal{U}_{\bar{m}_{0}, \bar{n}_{0}} d \mathscr{U}_{\bar{m}_{1}, \bar{n}_{1}} d \mathscr{U}_{\bar{m}_{2}, \bar{n}_{2}}=(2 \pi i)^{2} \mathscr{U}_{\bar{m}_{0}, \bar{n}_{0}}\left(\bar{m}_{1} \bar{n}_{2}-\bar{m}_{2} \bar{n}_{1}\right) \mathscr{U}_{\bar{m}_{1}, \bar{n}_{1}} \mathscr{U}_{\bar{m}_{2}, \bar{n}_{2}} \otimes \epsilon_{1} \wedge \epsilon_{2}
$$

The character of the cycle is given by

$$
\int \mathscr{U}_{\bar{m}_{0}, \bar{n}_{0}} d \mathscr{U}_{\bar{m}_{1}, \bar{n}_{1}} d \mathscr{U}_{\tilde{m}_{2}, \bar{n}_{2}}=(2 \pi i)^{2}\left(\bar{m}_{1} \bar{n}_{2}-\bar{m}_{2} \bar{n}_{1}\right) \tau\left(\mathscr{U}_{\tilde{m}_{0}, \bar{n}_{0}} d \mathscr{U}_{\bar{m}_{1}, \bar{n}_{1}} d \mathscr{U}_{\bar{m}_{2}, \bar{n}_{2}}\right) .
$$

Now notice that

$$
\mathscr{U}_{\tilde{m}_{0}, \bar{n}_{0}} \mathscr{U}_{\bar{m}_{1}, \bar{n}_{1}} \mathscr{U}_{\tilde{m}_{2}, \bar{n}_{2}}=\exp \left(2 \pi i \alpha\left(-\bar{n}_{0} \bar{m}_{1}-\bar{n}_{1} \bar{m}_{2}-\bar{n}_{0} \bar{m}_{2}\right)\right) \mathscr{U}_{\bar{m}_{0}+\bar{m}_{1}+\bar{m}_{2}, \bar{n}_{0}+\bar{n}_{1}+\bar{n}_{2}},
$$

and $\tau\left(\mathscr{U}_{m, n}\right)=\delta_{m, 0} \delta_{n, 0}$, so the result will be zero unless

$$
\bar{m}_{0}+\bar{m}_{1}+\bar{m}_{2}=0=\bar{n}_{0}+\bar{n}_{1}+\bar{n}_{2}
$$


and when this holds the above expression reads

$$
\int \mathscr{U}_{\bar{m}_{0, \bar{n}_{0}}} d \mathscr{U}_{\tilde{m}_{1}, \bar{n}_{1}} d \mathscr{U}_{\bar{m}_{2}, \bar{n}_{2}}=(2 \pi i)^{2}\left(\bar{m}_{1} \bar{n}_{2}-\bar{m}_{2} \bar{n}_{1}\right) e^{2 \pi i \alpha\left(\tilde{n}_{1} \bar{m}_{1}+\bar{n}_{2} \bar{m}_{1}+\bar{n}_{2} \bar{m}_{2}\right)}
$$

So we've established that

$$
\begin{aligned}
\tau(0,1,2) & =-\pi i \operatorname{Trace}\left(\epsilon F\left[F \mathscr{U}_{\tilde{m}_{0}, \tilde{n}_{0}}\right]\left[F, \mathscr{U}_{\tilde{m}_{1}, \tilde{n}_{1}}\right]\left[F, \mathscr{U}_{\tilde{m}_{2}, \tilde{n}_{2}}\right]\right) \\
& =\int \mathscr{U}_{\tilde{m}_{0, n_{0}}} d \mathscr{U}_{\tilde{m}_{1}, \tilde{n}_{1}} d \mathscr{U}_{\tilde{m}_{2}, \tilde{n}_{2}} .
\end{aligned}
$$

That is, the characters of the cycle $\left(\Omega, d, \int\right)$ defined above and the canonical cycle arising from the $p>2$-summable Fredholm module $(\mathscr{H}, F)$ are identical.

\section{§ 5.3. The Chern Number as an Analytical Index}

Now recall that Fredholm modules play the (non-commutative) role of elliptic operators, so we'd expect a canonical pairing of the Fredholm module over $A_{\alpha}^{\infty}$ found above with $K_{0}\left(A_{\alpha}^{\infty}\right)$. Now any class in $K_{0}\left(A_{\alpha}^{\infty}\right)$ has a representative projection in $A_{\alpha}^{\infty}$ [9], so consider $e \in \operatorname{Proj}\left(A_{\alpha}^{\infty}\right)$ and

$$
\begin{aligned}
T: e \mathscr{H}^{+} & \rightarrow e \mathscr{H}^{-} \\
\xi & \mapsto e F \xi .
\end{aligned}
$$

Then $T$ is the required Fredholm operator: an inverse modulo $\mathscr{K}$ is provided by

$$
\begin{aligned}
T^{\prime}: e \mathscr{H}^{-} & \rightarrow e \mathscr{H}^{+} \\
\eta & \mapsto e F \eta,
\end{aligned}
$$

for note that

$$
\begin{aligned}
\left(1-T T^{\prime}\right) \eta & =(1-e F e F) \eta \\
& =(e-e(e F+j) F e) \eta \text { for } j \in \mathscr{L}^{p}(\mathscr{H}),
\end{aligned}
$$

because $[F, e] \in \mathscr{L}^{p}(\mathscr{H})$ and $e \eta=\eta$. Thus we have

$$
\begin{aligned}
\left(1-T T^{\prime}\right) \eta & =\left(e-e^{2} F^{2}-j F e\right) \eta \\
& =-j F \eta
\end{aligned}
$$

and since $\mathscr{L}^{p}(\mathscr{H})$ is an ideal, $-j F \in \mathscr{L}^{p}(\mathscr{H}) \subset \mathscr{K}$. That is, $\left(1^{-}-T T^{\prime}\right) \in \mathscr{K}\left(\mathscr{H}^{-}\right)$, and similarly $\left(1^{+}-T^{\prime} T\right) \in \mathscr{K}\left(\mathscr{H}^{+}\right)$, and so $T$ is a Fredholm operator. The index 
of $T$ is thus integral, and is the Chern number corresponding to the projection $e$. That is,

$$
\frac{1}{2 \pi i} \tau\left(e\left[\delta_{1}(e), \delta_{2}(e)\right]=\frac{1}{2 \pi i} \tau(e d e d e)=\operatorname{ind}(T) \in \mathbb{Z}\right.
$$

Appendix: Normalization of $\mathscr{H}^{+}$

We briefly justify the statement contained in the text of this section that the lack of normalization of the $\Theta_{m, n}^{+} \in \mathscr{H}^{+}$does not affect the result of the calculations performed above. Reinstalling the normalization conditions, the basic relationships change as follows:

$$
\begin{aligned}
(\bar{\partial}+\epsilon) \Theta_{m, n}^{+} & =|i(m+i n)+\epsilon| \Theta_{m, n}^{-} \\
(\bar{\partial}+\epsilon)^{-1} \Theta_{m, n}^{+} & =\frac{\Theta_{m, n}^{+}}{|i(m+i n)+\epsilon|} .
\end{aligned}
$$

These give us

$$
\begin{aligned}
& {\left[F, \mathscr{U}_{\bar{m}, \bar{n}}\right] \Theta_{m, n}^{+}=|i \mathbf{m}+\epsilon| \exp (\pi i \alpha(\bar{m} n-m \bar{n}+\bar{m} \bar{n}))\left\{\frac{i((\mathbf{m}+\overline{\mathbf{m}}) \epsilon}{i \mathbf{m}+\epsilon}-1\right\} \Theta_{m+\bar{m}, n+\bar{n}}^{-}} \\
& {\left[F, \mathscr{U}_{\bar{m}, \bar{n}}\right] \Theta_{m, n}^{-}=\frac{-\exp (\pi i \alpha(\bar{m} n-m \bar{n}+\bar{m} \bar{n}))}{|i(\mathbf{m}+\overline{\mathbf{m}})+\epsilon|} \frac{i \overline{\mathbf{m}}}{i \mathbf{m}+\epsilon} \Theta_{m+\bar{m}, n+\bar{n} .}^{+}}
\end{aligned}
$$

In the calculation of $\left(F\left[F, \mathscr{U}_{\tilde{m}_{0}, \tilde{n}_{0}}\right]\left[F, \mathscr{U}_{\tilde{m}_{1}, \tilde{n}_{1}}\right]\left[F, \mathscr{U}_{\tilde{m}_{2}, \tilde{n}_{2}}\right]\right)$ then, the change reduces to

$$
\frac{1}{\left|i\left(\mathbf{m}+\mathbf{m}_{0}+\mathbf{m}_{1}+\mathbf{m}_{2}\right)+\epsilon\right|}|i \mathbf{m}+\epsilon|
$$

The two terms cancel upon application of the trace (under which only terms with $\bar{m}_{0}+\bar{m}_{1}+\bar{m}_{2}=0=\bar{n}_{0}+\bar{n}_{1}+\bar{n}_{2}$ survive). That is, the character is unaffected by the normalization constants.

\section{References}

[1] Anderson, J. and Paschke, W., The rotation algebra, Houston J. Math., 15 No.1, (1989), $1-26$.

[2] Auslander, L. and Moore, C., Unitary representations of solvable Lie groups, Mem. A.M.S., 62 (1966).

[3] Bellissard, J., K-theory of $C^{*}$-algebras in solid state physics, in Statistical Mechanics and Field theory, Mathematical aspects, Dorlas, T. C., Hugenholtz, M. N., Winnink, M. (eds), Lecture Notes in Physics, 257, Springer, Berlin, Heidelberg, New York (1986).

[4] . Ordinary quantum Hall effect and non-commutative cohomology, in Localization in Disordered Systems, Weller, W., Ziesche, P. (eds), Teubner, Leipzig, 1987.

[5] Bellissard, J., van Elst, A. and Schulz-Bades, H., The noncommutative geometry of the quantum Hall effect, J. Math. Phys., 35 (10) (1994), 5375-5451. 
[6] Bellissard, J. and Simon, B., Cantor spectrum for the almost Mathieu equation, J. Func. Anal., 48 (1982), 408-419.

[7] Carey, A, and Philips, J., Algebras almost commuting with Clifford algebras in a $\mathrm{II}_{\infty}$ factor, K-theory, 4 (1991), 445-478.

[8] Choi, M.-D., Elliott, G. and Yui, N., Gauss polynomials and the rotation algebra, Invent. Math., 99(1990), 225-246.

[9] Connes, A., A survey of foliation and operator algebras, in Proceedings of Symposia in Pure Mathematics, 38, A.M.S., Providence, RI, (1982).

[10] _ C $C^{*}$-algebres et géométrie différentielle, C. R. Acad.Sci.Paris, 290(1980), 599-604.

[11] Non-commutative differential geometry, Publ. Math. IHES, 62(1986), 257 - 360.

[12] Cycon, H. L., Simon, B. and Beiglbock, E. (eds), Schrödinger operators with applications to quantum mechanics and global geometry, texts and monographs in physics, SpringerVerlag, Berlin, New York (1987).

[13] Elliott, G., Gaps in the spectrum of an almost periodic Schrödinger operator, C. R. Math. Rep. Acad. Sci. Canada, 4(1982), 255-259.

[14] _ Gaps in the spectrum of an almost periodic Schrödinger operator II, in Geometric Methods in Operator Algebras, Araki, H., Effros, E. G. (eds), Pitman Research Notes in Math. Series 123, Longman, London (1986).

[15] Fradkin, E. and Kohmoto, M., Quantum Hall effect and geometrical localization of electrons on lattices, Phys. Rev. B, 35(1987), 6017-6023.

[16] Green, P., The local structure of twisted covariance algebras, Acta Math., 140(1978), 191-250.

[17] _ The structure of imprimitivity algebras, J. Func. Anal., 36(1980), 88-104.

[18] Grossman, A., Momentum-like constants of motion, in 1971 Europhysics Conference on Statistical Mechanics and Field Theory, Haifa, Sen, R. N., Weil, C. (eds), Halsted, New York, 1972.

[19] Hadju, J., Janßen, M. and Viehweger, O., Kubo Hall conductivity on a finite cylinder and the integer quantum Hall effect, $Z$. Phys. B, 66(1987), 433-439.

[20] Høegh-Krohn, R., Skjelbred, T., Classification of $C^{*}$-algebras admitting ergodic actions of the two-dimensional torus, J. Reine Angew. Math., 328(1981), 1-8.

[21] Mackey, G. W., Unitary representations of group extensions I, Acta Math., 99(1958), 265-311.

[22] Packer, J., K-theoretic invariants for $C^{*}$-algebras associated to transformations and induced flows, J. Func. Anal., 67(1986), 25-59.

[23] Packer, J. A. and Raeburn, I., On the structure of twisted group C*-algebras, Uni. N.S.W. preprint, 1989.

[24] Twisted crossed products of $C^{*}$-algebras, Math. Proc. Camb., Phil. Soc., 106(1989), $293-311$

[25] Reed, M. and Simon, B., Methods of modern mathematical physics, II , Academic Press, New York, 1972-1979.

[26] Rieffel, M. A., Applications of strong Morita equivalence to transformation group $C^{*}$-algebras, in Proceedings of Symposia in Pure Mathematics, 38, A.M.S., Providence, RI, (1982).

[27] $\quad C^{*}$-algebras associated with irrational rotations, Pac. J. Math., 93(1981), 415419.

[28] _ Morita equivalence for operator algebras, in Proceedings of Symposia in Pure Mathematics, 38, A.M.S., Providence, RI, (1982).

[29] Strong Morita equivalence of certain transformation group $C^{*}$-algebras, Math. Ann., 222(1976), 7-22.

[30] Ruskai, M.B., Inequalities for traces on von Neumann algebras, Comm. Math. Phys., 26 (1972), 280-289.

[31] Středa, P., Theory of quantised Hall conductivity in two dimensions, J. Phys. C,15 (1982), L717-L721.

[32] Shubin, M., Discrete magnetic Laplacian, Comm. Math. Phys., 164 (1994), 259-275. 
[33] Thouless, D., Quantum Hall effect in 2-d periodic potentials, Phys. Rep., 110(1984), $279-291$.

[34] Xia, J., Geometrical invariants of the quantum Hall effect, Comm. Math. Phys., 119 (1988), $29-50$. 